\title{
Cloud and DNI nowcasting with MSG/SEVIRI for the optimized operation of concentrating solar power plants
}

\author{
Tobias Sirch $^{1}$, Luca Bugliaro ${ }^{1}$, Tobias Zinner ${ }^{2}$, Matthias Möhrlein ${ }^{3}$, and Margarita Vazquez-Navarro ${ }^{1}$ \\ ${ }^{1}$ Deutsches Zentrum für Luft- und Raumfahrt, Institut für Physik der Atmosphäre, Oberpfaffenhofen, Germany \\ ${ }^{2}$ Meteorologisches Institut, Ludwig-Maximilians-Universität, Munich, Germany \\ ${ }^{3}$ Nowcast GmbH, Albert-Roßhaupter-Str. 43, Munich, Germany \\ Correspondence to: Tobias Sirch (tobias.sirch@dlr.de)
}

Received: 22 July 2016 - Published in Atmos. Meas. Tech. Discuss.: 21 September 2016

Revised: 22 December 2016 - Accepted: 22 December 2016 - Published: 2 February 2017

\begin{abstract}
A novel approach for the nowcasting of clouds and direct normal irradiance (DNI) based on the Spinning Enhanced Visible and Infrared Imager (SEVIRI) aboard the geostationary Meteosat Second Generation (MSG) satellite is presented for a forecast horizon up to $120 \mathrm{~min}$. The basis of the algorithm is an optical flow method to derive cloud motion vectors for all cloudy pixels. To facilitate forecasts over a relevant time period, a classification of clouds into objects and a weighted triangular interpolation of clear-sky regions are used. Low and high level clouds are forecasted separately because they show different velocities and motion directions. Additionally a distinction in advective and convective clouds together with an intensity correction for quickly thinning convective clouds is integrated. The DNI is calculated from the forecasted optical thickness of the low and high level clouds. In order to quantitatively assess the performance of the algorithm, a forecast validation against MSG/SEVIRI observations is performed for a period of 2 months. Error rates and Hanssen-Kuiper skill scores are derived for forecasted cloud masks. For a forecast of $5 \mathrm{~min}$ for most cloud situations more than $95 \%$ of all pixels are predicted correctly cloudy or clear. This number decreases to $80-95 \%$ for a forecast of $2 \mathrm{~h}$ depending on cloud type and vertical cloud level. Hanssen-Kuiper skill scores for cloud mask go down to $0.6-0.7$ for a $2 \mathrm{~h}$ forecast. Compared to persistence an improvement of forecast horizon by a factor of 2 is reached for all forecasts up to $2 \mathrm{~h}$. A comparison of forecasted optical thickness distributions and DNI against observations yields correlation coefficients larger than 0.9 for $15 \mathrm{~min}$ forecasts and around 0.65 for $2 \mathrm{~h}$ forecasts.
\end{abstract}

\section{Introduction}

Availability of energy power plays a central role in society and its economical evolution. Among the renewable energy sources, concentrating solar power (CSP) systems have a great potential since they combine electricity production with a storage capacity. By means of mirrors the incoming solar irradiance is concentrated, heating a fluid and driving a heat engine. The used technologies are parabolic trough, solar power tower, Fresnel reflectors and dish Stirling. In case of low insolation the electricity production is taken over by a fuel, e.g., gas. The operation of such solar power plants is challenging since the thermodynamic properties of the heated fluid are difficult to control, for instance when the CSP plant is only partially illuminated by the Sun or when insolation is strongly variable over time ranges of a few minutes to a few hours.

The fuel of solar power plants is direct normal irradiance (DNI). The main source of DNI spatiotemporal variability is cloudiness due to its intrinsic spatiotemporal inhomogeneity and to the fact that already thin clouds can reduce DNI to unusable levels for CSP. Further factors that affect DNI are aerosols and, to a lesser extent, water vapor and ozone (Gueymard, 2012). Thus, the knowledge and the prediction of cloud properties for the derivation of DNI is essential for the optimization of the CSP operation strategy because for day-ahead and intra-day electricity markets the electricity production must be announced to the market operator and deviations from the production schedule may lead to deviation penalties. Kraas et al. (2013) show the economic merits of a forecasting system for day-ahead forecasts for CSP, 
which reduces penalties by $47.6 \%$ compared to a persistence model. The persistence model (the cloud distribution observed at the forecast starting point is assumed to stay unchanged during the entire forecast time) is the simplest forecast model and works well for periods of low cloud variability and obviously for clear-sky cases. Of course, the accuracy of persistence models is reduced substantially when the variability increases. Therefore other methods are used based on data from various sources depending on the forecast horizon. For the prediction of solar irradiance 1 or 2 days ahead numerical weather prediction (NWP) models are used (Marquez and Coimbra, 2011), which provide better results when combined with artificial neural networks (ANNs) (Gonzalez et al., 2010). However, for short-term forecasts up to $6 \mathrm{~h}$ NWP models are not well suited and satellite-based methods come into play: Perez et al. (2013) and Lorenz et al. (2012) show that below a forecast horizon of 5-6 h, forecasting methods of NWP models have a lower accuracy compared to satellite-based algorithms (Eissa et al., 2013; Marquez and Coimbra, 2013). Other approaches deal with the detection and tracking of cloud patterns with satellite data - a challenging task due to the nonlinearity in atmospheric motion. They range from standard pattern recognition techniques (Bolliger et al., 2003; Wu, 1995; Wu et al., 1997; Schmetz et al., 1993a) to multichannel correlation-relaxation labeling (Evans, 2006). Geostationary satellites usually provide multispectral images with a temporal resolution between 5 and 30 min that can be used for determining the motion of clouds and their properties. However, their temporal and spatial resolution (on the order of kilometers) of satellite images are inappropriate for accurate forecasts of clouds for the next few minutes at particular (power plant) sites. This lack can be overcome by using local high-frequency imagecapturing devices, such as total sky imagers (Chu et al., 2013; Wacker et al., 2015) or other ground sensors (e.g., pyranometers, Bosch and Kleissl, 2013).

Convective clouds are of particular interest for society due to the high precipitation rates that are often connected to them. Because of their rapid development they increase the error in any forecast. Therefore it is reasonable to treat convective and advective clouds separately and to investigate the development of convective cells, which is a challenging task. During the last decades a large number of different cloud nowcasting approaches have been developed, most of them with a strong focus on thunderstorms. These techniques are using near real-time information from radars, e.g., CONRAD (Lang, 2001) and TRT (Hering et al., 2004), and passive imagers, e.g., Bolliger et al. (2003), Zinner et al. (2008) or Feidas and Cartarlis (2005), or a combination of both (Henken and Schmeits, 2011). There is also an effort to combine the advantages of radar data with lightning data (Steinacker et al., 2000) or additional numerical models (Pierce et al., 2000), but these methods have the disadvantage that only these areas can be observed where the used instruments are sited.
This publication presents a novel nowcasting algorithm based on satellite data from the Spinning Enhanced Visible and Infrared Imager (SEVIRI) aboard Meteosat Second Generation (MSG) for all clouds with a focus on clouds which are relevant for CSP generation. With its high repetition rate of $15 \mathrm{~min}$, its spatial sampling distance of $3 \mathrm{~km}$ and the availability of 12 spectral channels, this sensor is very well suited for the determination and forecast of cloud optical properties to be used to derive DNI since clouds are highly variable in space and time. Our method focuses on forecast times from 5 to $120 \mathrm{~min}$. It exploits an optical flow algorithm to determine atmospheric motion vectors for every pixel. The starting point is represented by the optical thickness of clouds that are first split up into two (vertically overlapping) layers in order to take care of different velocities of upper level and low level clouds. To reduce the turbulent character of the atmospheric motion field on small scales, rendering long-range forecasts impossible, cloud subsets are defined as rigid objects that move with time. Convection cannot be forecasted adequately this way, but our approach considers dissipating convective clouds, where extended anvils are produced that can live for many hours and have an important impact on DNI. DNI itself is eventually computed from the cloud optical thickness forecast. A validation against MSG/SEVIRI observations is shown at the end.

After a description of the satellite instrument MSG/SEVIRI and the cloud detection the analysis algorithms needed for our forecast method including the optical flow procedure are presented (Sect. 2). In Sect. 3 the nowcasting algorithm is described followed by its validation in Sect. 4.

\section{Instrument and tools}

MSG is a series of European geostationary satellites operated by EUMETSAT. Their primary mission is the observation of weather phenomena on the Earth's full disk with the SEVIRI imager. It consists of three channels in the visible, one in the near infrared and eight in the infrared spectral range with a sampling distance of $3 \mathrm{~km}$ at the sub-satellite point (Schmetz et al., 2002). Additionally a broadband high-resolution visible (HRV) channel is integrated, which covers only a part of the Earth's full disk with a higher spatial resolution of $1 \mathrm{~km}$ at the sub-satellite point. The usual repetition rate of $15 \mathrm{~min}$ is reduced to $5 \mathrm{~min}$ in rapid-scan mode for a part of the disk.

Optical, micro- and macrophysical properties of clouds are important parameters for the modeling of radiation-cloud interactions. Thus, their determination plays an important role for the computation of surface radiation, including DNI. The cloud detection and analysis algorithms used in this work are presented in the following two sections (Sect. 2.1 and 2.2). In Sect. 2.3 an optical flow method for the determination of cloud motion fields is described. 


\subsection{Cloud classification and cloud optical properties}

For thin ice clouds the "Cirrus Optical properties derived from CALIOP and SEVIRI during day and night" (COCS; Kox et al., 2014) algorithm is used. It is a back-propagation neural network, which is trained with collocated products of the depolarization-lidar CALIOP (Cloud-Aerosol Lidar with Orthogonal Polarization) aboard the Cloud-Aerosol Lidar and Infrared Pathfinder Satellite Observations (CALIPSO) and brightness temperatures as well as brightness temperature differences of MSG/SEVIRI. The COCS algorithm provides optical thickness (denoted by $\tau_{\mathrm{COCS}}$ in the following) and cloud top height for the highest ice cloud layer. In a validation study against airborne high spectral resolution lidar measurements (Kox et al., 2014), COCS detected $80 \%$ of the cirrus clouds with optical thickness 0.2 and its detection efficiency increased for higher optical thicknesses. For optical thickness 0.1 COCS detected still $50 \%$ of the cirrus clouds observed by CALIPSO. The false alarm ratio amounted to $2.6 \%$ for all measured cirrus clouds. It is very robust for small optical thicknesses above 0.1 up to a maximum of 2.5. Clouds with larger optical thickness cannot be penetrated by CALIOP (Winker et al., 2010).

In addition to COCS, APICS (Algorithm for the Physical Investigation of Clouds with SEVIRI; Bugliaro et al., 2011) is applied for the detection of liquid water clouds and thick cirrus. The APICS cloud detection is based on Kriebel et al. (2003) and consists of two groups of threshold tests applied to the solar SEVIRI channels. The first group detects a cloud when it is bright enough compared to the cloud-free reflectivity. The second is applied over sea and detects a cloud when the variability of the signal is higher than that of the cloudfree background (the sea surface reflectivity is supposed to be spatially homogeneous). A pixel is cloudy when at least one test gives a positive result; the pixel cloud phase can be liquid or ice.

A cloud top phase mask is produced by merging the results of the two algorithms. A cloud detected by COCS is an ice cloud, i.e., its cloud top is composed of ice. Clouds detected by APICS can be both ice and liquid water clouds. Thus, we assign all clouds detected by COCS to the ice phase, while a cloud that is detected by APICS but not by COCS is assigned to the liquid water phase.

Cloud optical properties are obtained the following way. COCS provides the optical thickness of the upmost cloud layer. Furthermore, for both cloud types (liquid water and ice), cloud optical thickness and effective radius are derived from two solar channels using APICS. In APICS, SEVIRI channels centered at 0.6 and $1.6 \mu \mathrm{m}$ are used based on the method by Nakajima and King (1990) and Nakajima and Nakajima (1995). The lookup tables required for this application have been computed with the radiative transfer model libRadtran (Mayer and Kylling, 2005; Emde et al., 2016) using a midlatitude standard atmosphere (Anderson et al., 1986), a typical continental aerosol load, a rural type aerosol in the boundary layer, background aerosol above $2 \mathrm{~km}$, springsummer conditions and a visibility of $50 \mathrm{~km}$ (Shettle, 1989). Surface albedo is taken from the temporally appropriate white sky MODIS albedo product MCD43C1 (Schaaf et al., 2002). For liquid water clouds, Mie cloud optical properties are assumed (spherical particles), while for ice clouds the parameterization by Baum et al. (2005) is used (a mixture of ice cloud habits as a function of ice crystal size). The optical thickness derived this way (denoted by $\tau_{\text {APICS, liq }}$ and $\tau_{\text {APICS, ice for liquid and ice water clouds respectively) ranges }}$ from 0 to 100 and refers to the entire atmospheric column. For ice clouds, $\tau_{\text {APICS, ice }}$ is usually higher than $\tau_{\mathrm{COCS}}$, which refers to the upmost ice cloud layer.

\subsection{Quickly developing convective clouds}

For the detection of convective clouds, procedures of the $\mathrm{Cb}$ TRAM algorithm (CumulonimBus TRacking And Monitoring; Zinner et al., 2008, 2013; Merk and Zinner, 2013) have been examined and exploited in this work. Cb-TRAM divides convection into three stages:

- convection initiation (stage 1)

- rapid cooling (stage 2)

- mature thunderstorm cells (stage 3).

In this work we consider only stage 3 , the detection of mature thunderstorm cells. It is limited to areas with a strong spatial roughness of the HRV, determined by the local standard deviation, combined with the brightness temperature difference of 6.2 and $10.8 \mu \mathrm{m}$. This is only valid for daytime. During night the HRV is replaced by a similar measure for the brightness temperature at $6.2 \mu \mathrm{m}$, which is less successful at confining the detection to the active updraft but includes large parts of the surrounding anvil and is independent of sunlight conditions; i.e., it produces smooth results in particular at sunrise, when the forecast is started. As these thinner clouds are most interesting for the derivation of DNI, the latter detection is used as part of the forecast algorithm in the following.

\subsection{Cloud motion fields}

The forecast rests upon an optical flow method determining a motion vector field from two consecutive images which is part of Cb-TRAM (Zinner et al., 2008). Unlike feature-based approaches, often used for the determination of atmospheric motion vectors for single objects/clouds in an image (e.g., Schmetz et al., 1993b), this method is pixel-based: instead of vectors only for interesting cloud patterns, a disparity vector field $\boldsymbol{V}(P)$ defined at each pixel position $P$ is derived.

Movements in the atmosphere take place on different scales reaching from microscale (few centimeters) to global scale $(10000 \mathrm{~km})$. These large-scale flows overlay the smallscale movements so that the determination of the disparity 

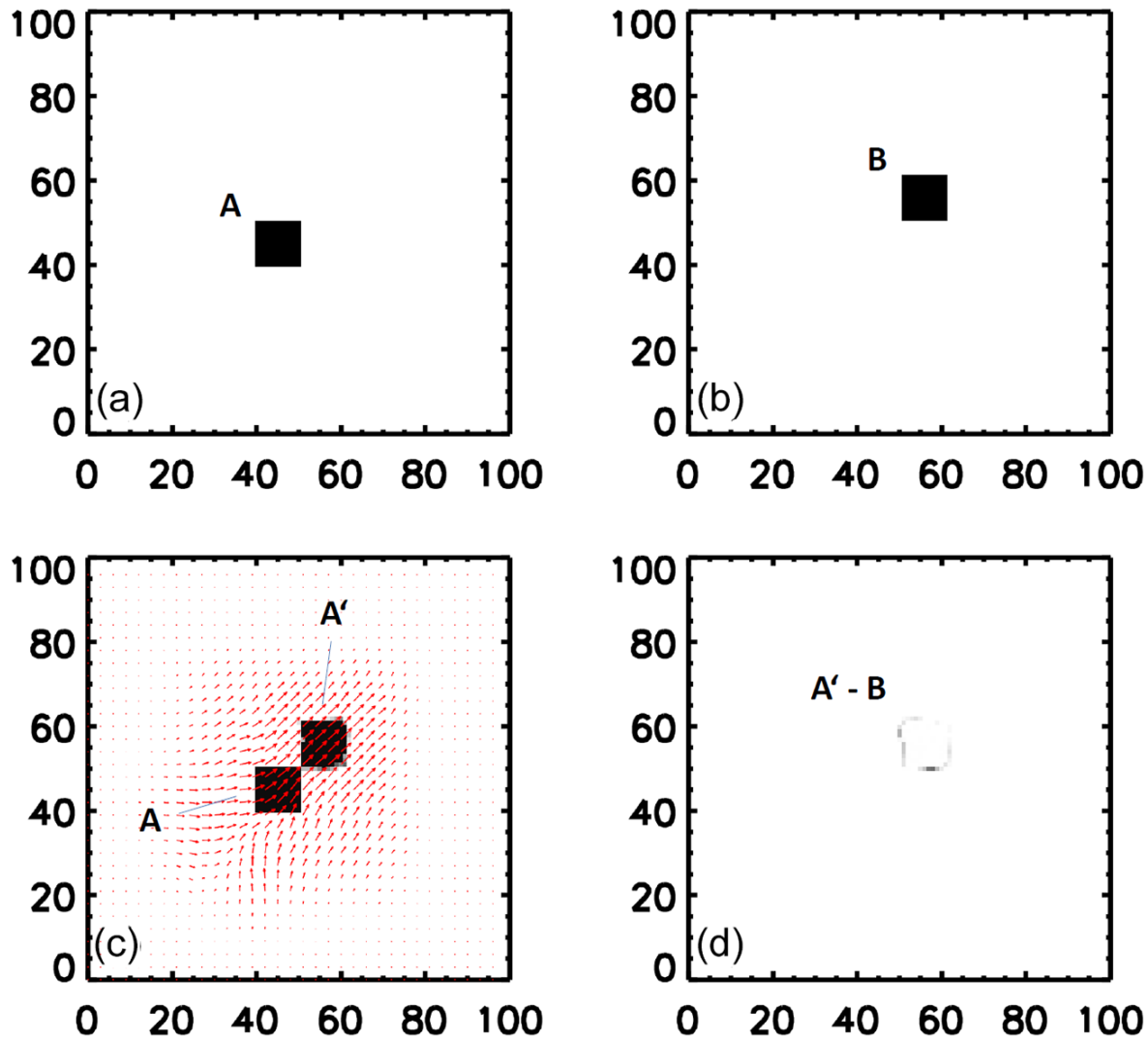

Figure 1. (a) Start image $A$. (b) Start image $B . A$ and $B$ are squares that have to be matched by the pyramidal matcher. (c) The final disparity vector field $\boldsymbol{V}$ is plotted on the start image $A$ and $A^{\prime}(P)=A\left(P-V_{A \rightarrow B}(P)\right)$ with (d) the remaining difference field $A^{\prime}-B$ after processing on all pyramid levels.

vector field for all scales is challenging. In order to take this into account the disparity vector fields are successively derived on different scales, starting from low resolution down to high resolution - a pyramidal scheme.

The procedure is described by means of an example for two images $A$ and $B$ (Fig. 1a, b) with a size of $n x=$ $100 \times n y=100$ pixels displaying two squares $(10 \times 10$ pixels). Similar structures in the images $A$ and $B$ are identified iteratively at different spatial scales, i.e., for all sub-sampling levels $l$ of the pyramidal approach with $M$ levels, starting with the topmost level with the roughest resolution:

1. Select the number of sub-sampling levels $N$ (e.g., $N=$ 2 for a pyramid with $M=N+1=3$ levels); this number depends on the size of the shifts that are expected.

2. Define the images $A_{M}=A$ and $B_{M}=B$, set $l=M-1$.

3. Start the iterative process.

3.1. Calculate the dimensions $n x_{l}=n x / 2^{l}$ and $n y_{l}=n y / 2^{l}$ of the given sub-sampling level $l\left(n x_{l} \times n y_{l}=25 \times 25\right.$ pixels for the topmost level, $n x_{l} \times n y_{l}=50 \times 50$ pixels for the second sub-level).
3.2. Resample the start images $A_{l+1}$ and $B_{l+1}$ to $n x_{l} \times n y_{l}$ to obtain $A_{l}$ and $B_{l}$.

3.3. Determine comparison images $A_{l, s}$ by shifting every pixel $P$ in image $A_{l}$ to $A_{l, s}=A_{l}\left(P+\Delta \boldsymbol{K}_{i, j}\right)$ by $\Delta \boldsymbol{K}_{i, j}=(i, j), i, j \in[-2,2]$, in both dimensions.

3.4. Identify the best fit between all possible $A_{l, s}$ and the target image $B_{l}$ through minimization of the squared difference of the intensities of $A_{l, s}$ and $B_{l}$ in a surrounding of each pixel: this results in the disparity vector field $V_{l}$ with dimensions $n x_{l} \times n y_{l}$.

3.5. To mitigate the impact of singular incorrect motion derivations and ensure physically realistic local flow fields, these initially integer displacements $\boldsymbol{V}_{\boldsymbol{l}}$ are smoothed over the local neighborhood of each pixel.

3.6. Blow up the resolution of $\boldsymbol{V}_{\boldsymbol{l}}$ to the original one $n x \times n y$ to obtain $\boldsymbol{V}_{l \text {, original }}$

3.7. Add the motion vectors obtained so far to $\boldsymbol{V}=$ $\sum_{i=l}^{N} V_{l, \text { original }}$ 
3.8. Warp the image $A$ with the disparity vector field $V$ to

$A_{l}(P)=A(P-V(P))$,

for every pixel position $P$. Notice that this equation implies that pixels $P$ in $A$ are not shifted with $V$ into $A_{l}$, but for every pixel $P$ in the forecast image $A_{l}$ a value from the starting image $A$ is assigned which can be found there at position $P-\boldsymbol{V}(P)$. So every pixel is allocated to a value and no information gaps (i.e., no "holes" in the image $A_{l}$ ) occur in the forecast. However, since $\boldsymbol{V}$ contains floating point values due to the smoothing in step 3.5, bilinear interpolation of $A$ in $x$ and $y$ is applied when performing Eq. (1). Thus, the warped image is only a "remapping" of the start image.

3.9. Reduce the value of $l$ by 1 and go back to step 3.1 if $l \geq 0$.

4. At the end of the iterative procedure, the refined disparity vector field $\boldsymbol{V}$ that has been obtained through successive addition of the results of all pyramidal levels provides the final disparity vector field $\boldsymbol{V}_{A \rightarrow B}$ in full resolution and its application to Eq. (1) the final warped image $A^{\prime}(P)=A_{l=0}(P)=A(P-V(P))$.

The refined disparity vector field $\boldsymbol{V}_{A \rightarrow B}$ in full resolution is shown in Fig. 1c. Notice that it is different from zero not only over the area defined by the initial image $A$ but also in the direct neighborhood. Due to that, disparity vectors are not always pointing from $A$ to $B$ but, outside of image $A$ and $B$, also in other directions. Figure 1c also shows the final warped image $A^{\prime}$. The displacement of image $A$ onto $B$ shows good results as the final remaining difference field (Fig. 1d) $A^{\prime}-B$ exhibits only small differences at the edges of the squares caused by the relaxation of the disparity analysis by smoothing.

For more details, technicalities and an additional example please see Zinner et al. (2008).

\section{Forecast algorithm}

In this section the forecast algorithm is described. It exploits the methods introduced in the previous section. First, a more advanced cloud classification is implemented that distinguishes two overlapping classes of clouds. Then, the pixelbased disparity vector field is determined for both cloud classes separately. Cloud objects are formed, based on optical thickness, and motion vectors are derived for these objects. After the assignment of motion vectors to cloud-free areas, clouds are warped to their new position with this motion vector field. An intensity correction is applied for rapidly thinning convective clouds. In a last step the DNI is calculated from the optical thickness.

\subsection{Step 1: cloud classification}

In the following, clouds are classified in SEVIRI images according to two criteria: The first one considers the cloud top phase and the vertical structure of clouds (Sect. 3.1.1); the second identifies a type of convective clouds particularly relevant for our application, dissipating convective clouds with a thinning anvil (Sect. 3.1.2).

\subsubsection{Cloud optical properties}

Low level and high level clouds are often observed to move in different directions at different velocities due to complex wind profiles in the atmosphere. In order to take this aspect into account, we aim at the separation of low and high level clouds and the generation of two forecasts, one for low level and one for high level clouds. However, using APICS and COCS applied to SEVIRI satellite data according to Sect. 2.1, this is only possible to some extent. A high ice cloud layer as detected by COCS might occur in the same pixel as a low level liquid water cloud. Optical thickness of the cirrus cloud is then well accounted for by the COCS result, while APICS provides an approximation of the total optical thickness of the upper ice cloud and the lower water cloud together. Inaccuracies are due to the fact that cloud optical thickness is always derived by APICS according to the given cloud top phase, when the atmospheric column consists of both liquid water and ice this assumption fails and the resulting optical thickness is only an approximation to the correct total optical thickness. Furthermore, the ice layer detected by COCS might be the upper layer of a vertically and optically much thicker cloud like a $\mathrm{Cb}$ (cumulonimbus). In this case, the total optical thickness of the cloud is most likely much larger than the COCS maximum value of 2.5 , and APICS can much better capture this aspect since its optical thickness is based on the reflectivity of the entire atmospheric column.

In general, the discrimination among all these cases and the determination of optical properties for all cloud layers is challenging using only passive satellite observations. Several approaches have been proposed (for instance, Joiner et al., 2010; Gonzalez et al., 2003; Huang et al., 2005; Baum et al., 1995). In this work, we want to forecast surface DNI, which becomes $\approx 0.1 \%$ of the original value for a slant optical thickness of 7 , as obtained by radiative transfer computations. To this end, all liquid water clouds usually reduce DNI to values far below the range interesting for CSP production due to their high optical thickness. Thus, accuracy in this range is not crucial. In case of thin cirrus, however, surface DNI is not zero and the accuracy of the ice cloud optical thickness is important as CSP, like parabolic troughs, is shut down when DNI $<200 \mathrm{~W} \mathrm{~m}^{-2}$, which corresponds to a vertical optical thickness of 2.

Qualitative indications contained in SEVIRI's spectral channels can be exploited to provide a reasonable differen- 
tiation between one layer and two layer cloud situations. For clarification an example is depicted in Fig. 2 for 7 April 2013. On this day a frontal zone is crossing the Iberian Peninsula. A SEVIRI false color composite (Fig. 2a) suggests the vertical structure of the clouds. The yellow colored cloud of the frontal zone consists of low, warm water clouds (i.e., a small blue component for which the inverted channel at $10.8 \mu \mathrm{m}$ is used). In other regions these clouds are overlaid by high thin ice clouds with low temperatures (blueish or violet colors). Over the Mediterranean Sea, east of Gibraltar, singlelayer ice clouds are observed. We exploit the differences between the APICS and COCS results for ice and liquid optical thickness to define two possibly overlapping classes of clouds called upper clouds and lower clouds that enable us to differentiate among these cases. The classification is summarized in Table 1 and explained in detail in the following.

Liquid water clouds identified following Sect. 2.1 are assigned to the lower cloud layer and their optical thickness is the APICS optical thickness. If APICS and COCS indicate a thin ice cloud ( $\left.\tau_{\text {APICS, ice }} \leq 2.5\right)$, the presence of an ice cloud without lower liquid cloud layers is assumed and optical thickness of COCS is assigned to the upper cloud layer (because COCS is assumed to be more accurate than APICS for thin cirrus clouds). For a cloud with ice top and APICS ice optical thickness larger than 2.5, the difference between the APICS ice optical thickness and the COCS optical thickness is investigated. If $\tau_{\text {APICS, ice }}-\tau_{\text {COCS }} \leq 2.3$, this is interpreted as possible deviation between two different methods providing results for the same cloud: please notice that if $\tau_{\mathrm{APICS}}$, ice is smaller than $\tau_{\mathrm{COCS}}$ then this condition is always fulfilled, while an upper limit to $\tau_{\mathrm{APICS}}$, ice is set here as $\tau_{\mathrm{COCS}}+2.3$; i.e., $\tau_{\mathrm{APICS}}$, ice can be at most approximately twice as large as $\tau_{\text {COCS }}$. Since COCS is supposed to be more accurate than APICS for thin cirrus (and for the sake of a "consistent" treatment of thin ice clouds), COCS optical thickness is selected for the upper cloud while the lower cloud optical thickness is set to zero. When in contrast APICS retrieves an ice optical thickness larger than 2.5 (the upper limit of COCS) and the difference between APICS and COCS is larger than 2.3 ( $\tau_{\text {APICS, ice }}-\tau_{\text {COCS }}>2.3$ ), this difference is assumed to have physical reasons due to the presence of a lower cloud layer. The situation encountered here is thus either a thin ice cloud on top of a water cloud or a vertically extended cloud with ice, liquid or mixed-phase microphysics below the upper ice layer. In all cases the cloud is thick enough that DNI at the surface is diminished to below $10 \%$ of its top-of-atmosphere value. The correct optical thickness distribution between lower and upper cloud cannot be determined, we arbitrarily set the optical thickness of the lower cloud to $\tau_{\text {APICS, ice }}$ and that of the upper cloud to $\tau_{\text {COCS }}$. This ensures that the upper cloud is considered correctly in case of a thin cirrus on top of a low liquid water cloud. Then, when the upper and the lower clouds are moving in different directions and the Sun can shine through the thin cirrus to the ground, the most appropriate ice cloud op- tical thickness is used. In contrast, liquid cloud optical thickness is not important because it is usually so high that no DNI can illuminate the surface. For a $\mathrm{Cb}$-like cloud, this decision is again not crucial for our application since the cloud usually moves as a whole (i.e., lower and upper layer continue to overlap) and its total optical thickness is so high that DNI at the surface is always zero. For this classification, the threshold value of 2.3 used above has been determined empirically based on visual inspection of false color composites like the one shown in Fig. 2. This classification does not claim to be exhaustive and could be further optimized, e.g., by the use of CALIPSO/CALIOP lidar data. However, it has the advantage of being computationally fast and enables the detection of liquid water clouds below thin ice clouds and the discrimination between thin and thick ice clouds. It is now possible to follow such a cloud when it is shaded by the advection of a thin cirrus cloud as long as the cirrus is thin enough.

The results of the classification applied to Fig. 2a are provided in Fig. 2b, c, d. The yellow colored clouds (low warm clouds) are characterized by the blue colored region in the cloud phase mask shown in Fig. 2b, unless they are overlaid by high thin ice clouds (blueish colors in Fig. 2a), in which case they are detected as multiphase clouds in the cloud mask (red color). The green areas in Fig. 2b denote single-layer ice clouds. Cloud-free areas are depicted in white. Figure $2 \mathrm{c}, \mathrm{d}$ show the corresponding optical thickness for the upper and lower cloud layer derived by COCS and APICS according to the procedure described above and in Table 1.

\subsubsection{Convective clouds}

The focus of the presented forecast method is the accurate prediction of thin ice clouds since they modulate surface DNI in the relevant range for CSP. Often ice clouds are formed by convection. In contrast to most ice clouds that are mainly characterized by horizontal advection, convective clouds show a strong local vertical development. While during growth and maturity of convective cells large optical thickness values dominate and DNI at surface is negligible, anvil ice clouds formed during maturity can live much longer than the thunderstorm cloud itself during the decaying stage (Byers and Braham, 1948). Thus they can lead to large but isolated cirrus clouds that are indeed interesting for DNI. Considering that convection is stronger and more important at low latitudes, where the solar power potential is high too, the specific consideration of decaying convective clouds represents an important aspect.

For this reason, a third class of clouds is defined: we single out mature convective clouds using the stage 3 detection of the Cb-TRAM algorithm as discussed in Sect. 2.2. This classification is independent of the previous classification in lower and upper cloud layers (Sect. 3.1.1), but due to the nature of the convective life cycle $\mathrm{Cb}$-TRAM stage 3 nighttime detections turn out to always belong to the upper cloud layer. 
Table 1. Assignment of cloud optical thickness to two cloud classes called upper clouds and lower clouds. $\tau_{\text {APICS, liq }}$ is the APICS optical thickness for clouds with liquid cloud top phase, $\tau_{\text {APICS, ice }}$ is the APICS optical thickness for clouds with ice cloud top phase, $\tau_{\mathrm{COCS}}$ is the COCS ice optical thickness, $\tau_{\text {low }}$ is the optical thickness assigned to the lower clouds, and $\tau_{\text {up }}$ is the optical thickness assigned to the upper clouds.

\begin{tabular}{lll}
\hline Liquid water cloud, no ice cloud above & & \\
$\tau_{\text {APICS, liq }}>0 \vee \tau_{\text {COCS }}=0$ & $\tau_{\text {low }}=\tau_{\text {APICS, liq }}$ & $\tau_{\text {up }}=0$ \\
\hline Thin cirrus cloud, no water cloud below & & \\
$\tau_{\text {APICS, ice }} \leq 2.5 \vee \tau_{\text {COCS }}>0$ & $\tau_{\text {low }}=0$ & $\tau_{\text {up }}=\tau_{\text {COCS }}$ \\
\hline Thick cirrus cloud, no water cloud below & & \\
$\tau_{\text {APICS, ice }}>2.5 \vee\left(\tau_{\text {APICS, ice }}-\tau_{\text {COCS }}\right) \leq 2.3$ & $\tau_{\text {low }}=0$ & $\tau_{\text {up }}=\tau_{\text {COCS }}$ \\
\hline Multilayer cloud & & \\
$\tau_{\text {APICS, ice }}>2.5 \vee\left(\tau_{\text {APICS, ice }}-\tau_{\text {COCS }}\right)>2.3$ & $\tau_{\text {low }}=\tau_{\text {APICS, ice }}$ & $\tau_{\text {up }}=\tau_{\text {COCS }}$ \\
\hline
\end{tabular}
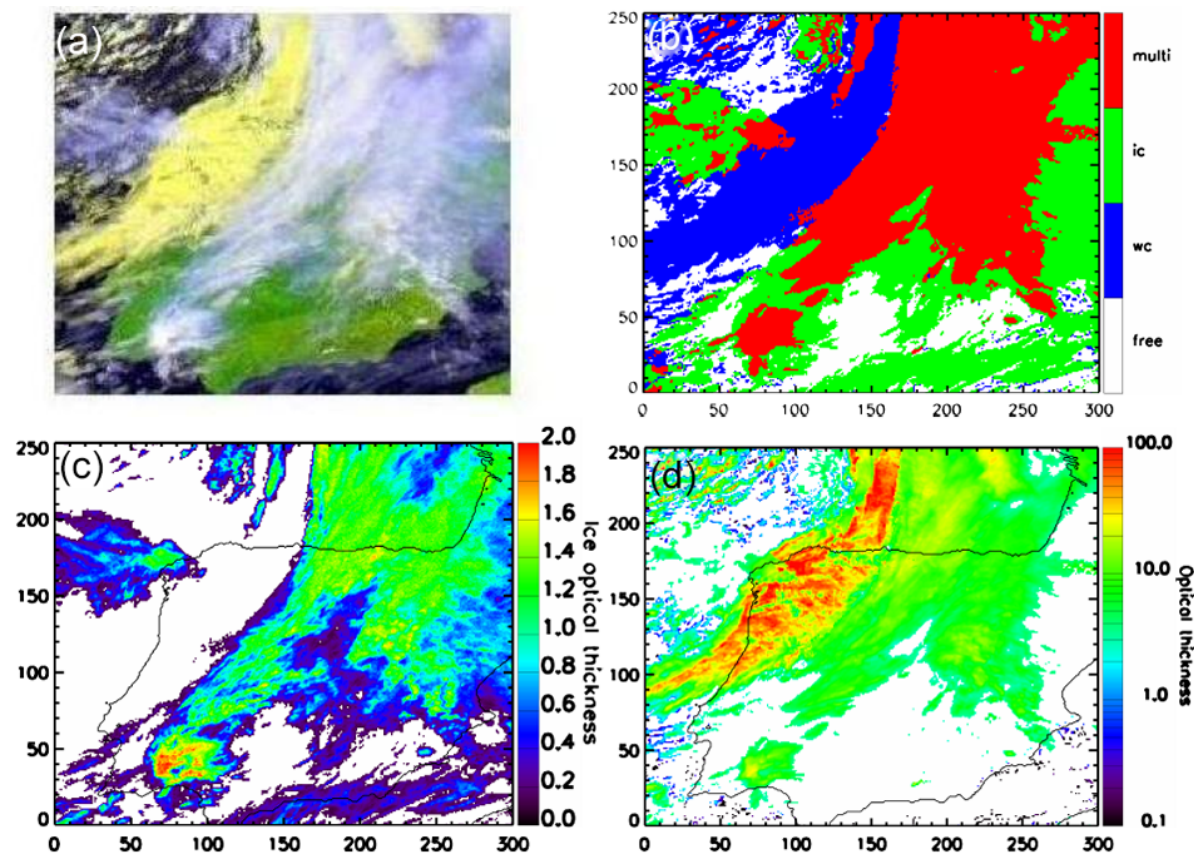

Figure 2. (a) False color composite (channels centered at 0.6, 0.8 and 10.8 $\mu \mathrm{m}$ ) for 7 April 2013, 13:15 UTC, for the Iberian Peninsula. (b) The cloud mask for this scene with ice clouds in green, water clouds in blue, multiphase clouds in red and cloud-free areas in black. The optical thickness for upper (c) and lower (d) clouds.

\subsection{Step 2: motion vectors}

Once clouds have been classified and cloud optical thickness has been determined (Sect. 3.1.1), lower and upper clouds can be considered separately; i.e., two forecasts are implemented, one for lower and one for upper clouds. Thus we proceed in the following way. First, the optical flow method described in Sect. 2.3 (often referred to as the matcher) is applied separately to lower and upper clouds, producing two independent "pixel-based" motion vector fields (Sect. 3.2.1). Second, for reasons that will become evident below, these two motion vector fields are averaged over specific cloud subsets (Sect. 3.2.2). Finally, motion vectors are provided for the cloud-free areas (Sect. 3.2.3).

\subsubsection{Pixel-based motion vectors}

In this first stage motion vector fields are derived for the optical thickness of lower clouds and upper clouds separately. Since convective clouds as defined in Sect. 3.1.2 are a subset of the upper clouds, they are not mentioned explicitly here since they do not play any role at this point. Note that in order to avoid edge effects one should match areas larger than the given region of interest: the selected area should be as large as to allow the observations of all clouds that will en- 
ter the region of interest during the time span needed for the forecast, in this case $120 \mathrm{~min}$.

There are two reasons for the use of the optical thickness as input parameter for the optical flow method: first, it is the quantity need for the calculation of DNI (see Sect. 3.5); second, the matcher works best if only the objects that are meant to move are matched against each other - in this case the cloud objects.

Forecasts are produced in forecast steps of $\Delta t_{\mathrm{f}}=5 \mathrm{~min}$ up to a forecast horizon of $120 \mathrm{~min}$. First, the disparity vector field $\boldsymbol{V}_{A \rightarrow B}$ between the initial images $A$ and $B$ separated by a time interval $\Delta t=15 \mathrm{~min}$ is determined by the pyramidal matcher with $N=3$ pyramidal sampling levels (see Sect. 2.3). Accordingly, the possible "search radius" is given by at least $2^{(N+2)}=32$ pixels, corresponding to an atmospheric motion of more than $360 \mathrm{~km} \mathrm{~h}^{-1}$ at midlatitudes for the operational SEVIRI scan mode with 15 min repetition time (see also Zinner et al., 2008). Then, a disparity vector field $V_{5 \text { min }}$ according to the length of the time step $\Delta t_{\mathrm{f}}$ is computed by multiplication of the disparity vector field $\boldsymbol{V}_{A \rightarrow B}$ by $d=\Delta t_{\mathrm{f}} / \Delta t=1 / 3$ :

$\boldsymbol{V}_{5 \min }=d \cdot \boldsymbol{V}_{A \rightarrow B}$.

The forecast image $F_{5}$ min for the lead time of $5 \mathrm{~min}$ is then produced according to Eq. (1) with the corresponding disparity vector field $V_{5 \mathrm{~min}}$ applied to the later initial image $B$ :

$F_{5 \min }(P)=B\left(P-V_{5 \min }(P)\right)$ for all pixels $P$.

Forecasts with longer lead times can be performed as well. For the next forecast step of $10 \mathrm{~min}$ the 2-D disparity vector field $\boldsymbol{V}_{5 \mathrm{~min}}=\left(u_{5 \mathrm{~min}}, v_{5 \mathrm{~min}}\right)$ is shifted with itself, i.e., its components are advected according to the cloud-air motion:

$u_{5 \min , \text { shifted }}(P)=u_{5 \min }\left(P-V_{5 \min }(P)\right)$

$v_{5 \text { min, shifted }}(P)=v_{5 \min }\left(P-V_{5 \min }(P)\right)$.

The shifted disparity vector field $V_{5 \text { min, shifted }}=$ $\left(u_{5 \text { min, shifted }}, v_{5 \text { min, shifted }}\right)=\boldsymbol{V}_{5 \min }\left(P-\boldsymbol{V}_{5 \min }(P)\right)=$ :

$\boldsymbol{s}^{(1)}(P)$ provides the information about the disparity vector field at the position where the pixels will be located according to the atmospheric flow after $5 \mathrm{~min}$. This vector is then added to $\boldsymbol{V}_{5 \text { min }}$ to produce $\boldsymbol{V}_{10 \text { min }}$ :

$\boldsymbol{V}_{10 \min }(P)=\boldsymbol{V}_{5 \min }(P)+\boldsymbol{s}^{(1)}(P)$

and

$F_{10 \min }(P)=B\left(P-V_{10 \min }(P)\right)$ for all pixels $P$.
This procedure is iterated for further time steps according to the general formula

$$
\begin{aligned}
& \boldsymbol{V}_{n \cdot 5 \min }(P)=\boldsymbol{V}_{(n-1) \cdot 5 \min }(P)+\boldsymbol{s}^{(n-1)}\left(P-\boldsymbol{s}^{(n-1)}(P)\right) \\
& F_{n \cdot 5 \min }(P)=B\left(P-\boldsymbol{V}_{n \cdot 5 \min }(P)\right), n \geq 2,
\end{aligned}
$$

where any $\boldsymbol{s}^{(n)}$ is determined recursively as

$\boldsymbol{s}^{(n)}(P)=\boldsymbol{s}^{(n-1)}\left(P-\boldsymbol{s}^{(n-1)}\right)$

$\boldsymbol{s}^{(1)}(P):=\boldsymbol{V}_{5 \min }\left(P-\boldsymbol{V}_{5 \min }(P)\right)$.

Physically, this approach means that the motion vector field $V_{5 \text { min }}$ is supposed to describe the atmospheric flow as it can be determined from the two initial images. The forecast procedure, Eq. (2), follows the atmospheric flow in steps of 5 min by evaluating $\boldsymbol{S}^{(n)}$ at the different positions a cloud-air parcel runs through with time.

To illustrate the result of this forecast procedure we consider the upper cloud layer from the example in Fig. 2. The optical thickness of these clouds is depicted in Fig. 3 for 13:00 UTC (a) and 13:15 UTC (c). The disparity vector field $\boldsymbol{V}=\boldsymbol{V}_{A \rightarrow B}$ obtained from these two images is also displayed in Fig. 3c using small arrows. For clarity only 1 out of 10 vectors is shown. Nonetheless, the cloud motion vectors still exhibit a strong horizontal variability, especially inside cloud regions and close to them. The large cloud field in the eastern part of the Iberian Peninsula is generally shifted towards east or northeast. However, motion vectors abruptly vary from one pixel to the next. The motion vector field is applied to the 13:15 UTC image (using Eq. 2) to produce a $1 \mathrm{~h}$ forecast (Fig. 3d). This forecast shows several deficiencies compared to the real cloud optical thickness observed at this time (Fig. 3b) for the following reason: the pyramidal matcher provides a detailed motion field representative only for changes during a (short) 15 min time period. Smallscale turbulence and changes produce a very variable disparity vector field (in direction and absolute value) not representative for a longer time period. As a consequence, cloud patterns dissolve into small patches within a short period of time, which does not correspond to reality as only the average larger-scale motions stay stable over longer periods.

\subsubsection{Object-based cloud motion vectors}

For the reason discussed above, an averaging procedure for the pixel-based cloud motion vectors is implemented. To this end, neighboring pixels with similar optical thickness are combined to objects. This procedure is called object classification and is applied separately to upper and lower cloud layers since they are forecasted separately. At this step, convective clouds are treated separately. This averaging procedure removes small-scale variability that is realistic at the moment of derivation but makes the forecast unstable.

For upper clouds, first each convective cell (Sect. 2.2) is classified as an individual object as prerequisite for the 

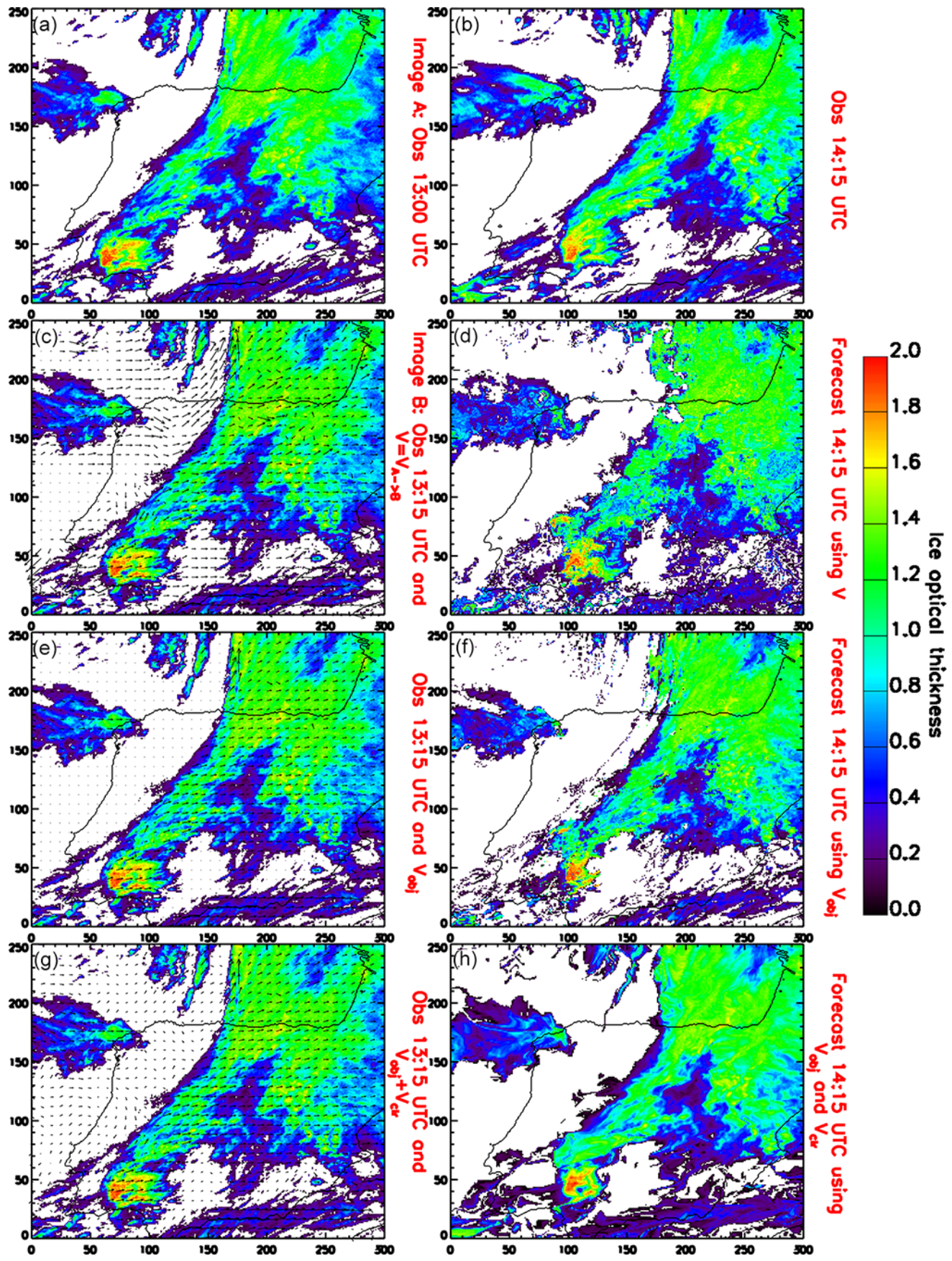

Figure 3. Illustration of the forecast of optical thickness for upper clouds for 7 April 2013 (a) and (c). Initial images A (13:00 UTC) and B (13:15 UTC) with the calculated pixel-based disparity vector field on top. (b) Upper cloud optical thickness at 14:15 UTC. (d) Pixelbased $1 \mathrm{~h}$ forecast (i.e., for 14:15 UTC) of upper cloud optical thickness. (e) Upper cloud optical thickness at 13:15 UTC with the calculated object-based disparity vector field $\boldsymbol{V}_{\text {obj }}$ on top. (f) Object-based $1 \mathrm{~h}$ forecast for 14:15 UTC of upper cloud optical thickness. (g) Upper cloud optical thickness at 13:15 UTC with the calculated object-based disparity vector field on top for cloudy areas $\boldsymbol{V}_{\text {obj }}$ and cloud-free areas $\boldsymbol{V}_{\text {clr }}$. (h) Object-based $1 \mathrm{~h}$ forecast for 14:15 UTC of upper cloud optical thickness including cloud-free motion vectors.

application of a specific procedure presented further down (Sect. 3.3). For the remaining part of the upper cloud layer, the optical thickness range $[0.1,2.5]$ is divided into eight classes with a bin size of 0.3 to create objects. An example for this object classification is depicted in Fig. 4. The upper cloud layer (left panel in Fig. 4), which does not contain any convective cell in this case, is separated into 39 objects (right panel in Fig. 4). Each object consists of all contiguous pixels 

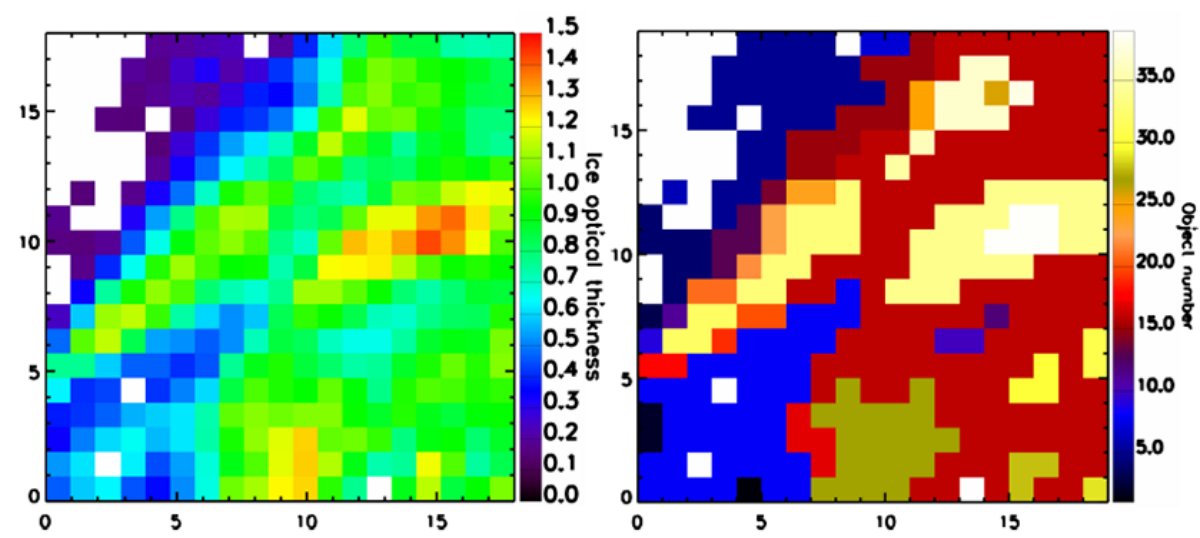

Figure 4. Upper cloud layer optical thickness extracted from the lower left part of Fig. 3c (left). Corresponding classification into 39 objects: pixels with the same color belong to the same object (right).

belonging to the same of the eight optical thickness classes. The size of the single objects varies strongly from 1 pixel to 50 pixels or more.

For the lower clouds the object classification is performed in a similar way: optical thickness in the range $[0,100]$ is divided into 10 intervals with a width of 10 .

Next, a mean motion vector is calculated for each object and this vector is assigned to every pixel in the object; i.e., the object moves as a whole during the forecasting procedure. The forecast image produced this way is called objectbased forecast. An example is shown in Fig. 3e, f for upper clouds. One can observe that the object-based cloud motion vector field $\boldsymbol{V}_{\text {obj }}$ is much smoother and points mainly to the east in the southern part and to the northeast in the northern part. The front position is well captured by the object-based forecast when compared to the observation. Comparing the object-based forecast (Fig. 3f) to the pixel-based (Fig. 3d), it can be seen that the front line stays much more stable in the object-based forecast and the isolated cloud to the west (pixel position between 0 and 150 in $x$ and between 150 and 200 in $y$ ) is moving as a whole and compares very well to the observation. However, the elongated cloud patches north of Spain (between pixel 100 and 150 in $x$ and above pixel 200 in $y$ ) cannot be forecasted well and still the edge of the forecasted cloud layer looks too patchy.

\subsubsection{Motion vectors for cloud-free areas}

As the motion vectors are derived from cloud optical thickness, the disparity vector field in the area between the clouds goes to zero (Fig. 3e). In case those cloud objects move into these regions, they stop. The thin line left of the front line and the squeezed cloud in the lower left corner (between pixel 50 and 150 in $x$ and between 0 and 100 in $y$ ) in Fig. 3f show this effect. This is partially compensated through the mentioned advection of the disparity vector field before its use in the forecast. The remaining effect, for forecasts over extended lead times, is further minimized if the cloud-free

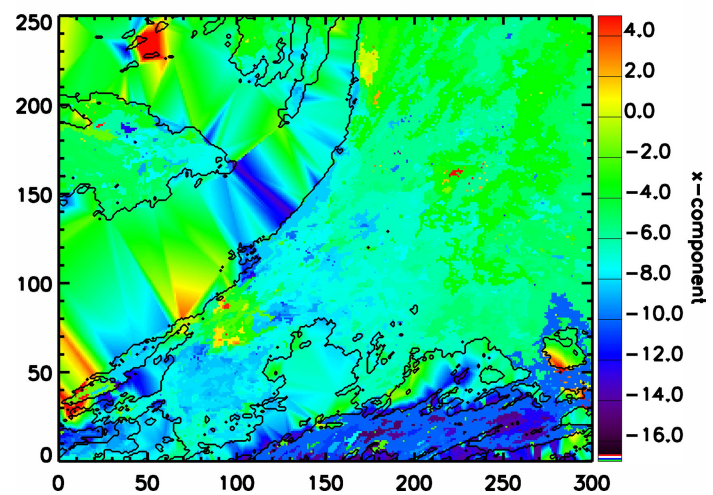

Figure 5. Delaunay triangulation for the $x$ component of the disparity vector field for upper clouds (black contours) returning a regular triangular grid of interpolated values between the clouds.

areas are filled with sensible motion estimates. The disparity vector field is divided into the object field for the clouds $\boldsymbol{V}_{\text {obj }}$ and the field $\boldsymbol{V}_{\text {clr }}$ for the clear-sky areas called background. A weighted triangular interpolation of the disparity vector field between clouds is applied. A Delaunay triangulation creates a triangle mesh for interpolation between single cloud-object-related vectors returning a regular grid of interpolated values. Delaunay triangulations avoid sliver triangles by maximizing the minimum angle of all the angles in the triangulation. Therefore, a relatively uniform field can be created. Values inside the triangles issue from a smooth quintic interpolation of the wind field. The method used for the triangulation is the divide-and-conquer algorithm from Lee and Schachter (1980). In Fig. 5, a weighted interpolation for the $x$ component of the disparity vector field is shown. In cloudy areas (black contour) the derived disparity vector field is used, while the interpolated values for the background field are calculated between the clouds (triangular shape). For forecast applications the values of the background field are limited to a range between -5 and 5 to avoid high gradi- 


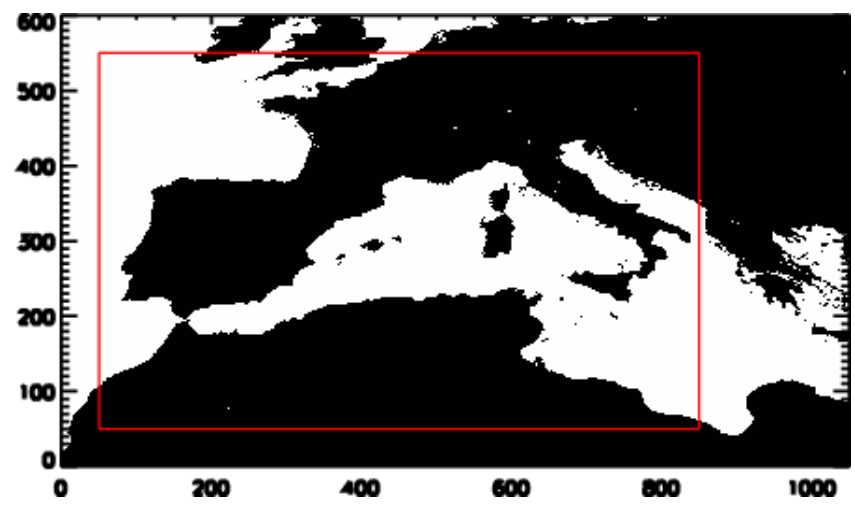

Figure 6. Domain used for the classification of decaying cells and for the validation presented in Sect. 4 (red square).

ents. The resulting disparity vector field (Fig. $3 \mathrm{~g}$ ) is significantly smoother than before (Fig. 3c).

\subsection{Step 3: intensity correction for quickly thinning convective clouds}

The pyramidal matcher (Sect. 2.3) can only predict the movement of the features in the images, i.e., rearrangement of values including divergence and convergence, but cannot create values in a given local area which could not be found, roughly speaking, within the "search radius" defined by the typical local wind-disparity vector (apart from the bilinear interpolation implemented in Eq. 1). This means that a local development of values of optical thickness is very limited in our case since the matcher is rather thought to detect object displacement and distortion. Neither in situ formation of cirrus clouds, which are particularly important for DNI, nor the time and place of convective initiation can be predicted. Once a cloud is observed its future evolution can be forecasted by continuation of the observed development: e.g., an increase in optical thickness in a cloud patch can be forecasted through disparity vectors, as far as it can be represented by pure growth of areas with values of optical thickness present in the source image. Values larger than the ones found in the local surrounding around the cloud patch in the source image cannot be provided in the forecast. As mentioned before, the decaying stage of convective cells is of interest for the purpose of DNI forecasting as thinning cirrus might allow an earlier recovery to DNI levels useful for CSP production (usually DNI $>200 \mathrm{~W} \mathrm{~m}^{-2}$ ). In contrast to the growing stage where, by no means, a nowcast of convective cell positions for the future $2 \mathrm{~h}$ is possible, for the decaying stage at least some useable initial information on the convective cloud is available. We found out that in this particular case, when convective cells start to decay leaving behind a thinning anvil cirrus layer, the temporal evolution of the cloud optical thickness can be reasonably well forecasted or at least improved with respect to the output of the matcher.

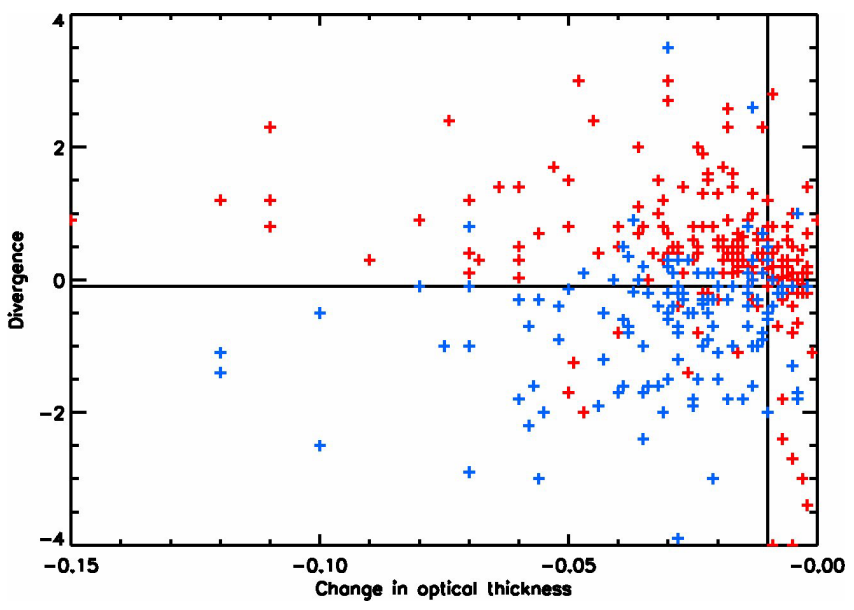

Figure 7. Distribution of the change in ice optical thickness in relation to the divergence with blue crosses denoting the decaying cells and red for the non-decaying cells.

To this end, quickly thinning convective clouds are first identified in satellite data and the successive evolution of their optical thickness, as far as it can be forecasted through disparity vectors, is then corrected to follow typical temporal patterns. Both the identification of these clouds and the determination of typical values for the temporal evolution have been developed based on 300 cells detected by Cb-TRAM (stage 3, mature cells, according to the classification presented in Sect. 3.1.2). They have been investigated manually in an area covering central and southern Europe including the western part of Mediterranean Africa with a size of $1050 \times 600$ pixels (Fig. 6) and for the time period April-June 2013. Cells were classified as decaying cells in cases where a decrease in optical thickness and a convergence of the anvil could be observed for the next time steps (temporal resolution $15 \mathrm{~min})$. The "divergence" $\operatorname{div}(\boldsymbol{V})$ is derived from the motion vector field $\boldsymbol{V}=(u, v)$ for each pixel:

$\operatorname{div}(\boldsymbol{V}):=\left(u_{\text {right }}-u_{\text {left }}\right)+\left(v_{\text {above }}-v_{\text {below }}\right)$

with the motion vector components $(u$ and $v)$ of the four neighboring pixels above, below, right and left of the pixel under investigation. A positive value denotes a divergence, while a negative value indicates a convergence. Figure 7 shows the distribution of the change in upper cloud layer optical thickness from one time step to the next, averaged over an entire convective cell in relation to the average divergence of the given cell. The blue crosses denote cells which were found to be in decaying stage by eye and red ones for non-decaying cells. Obviously most blue crosses concentrate in a region with divergence smaller than -0.1 (horizontal line) and below a change in optical thickness of -0.01 (vertical line). As only a few red crosses for the apparently nondecaying cells lie in this area, these object-averaged parameters can be used in an automated procedure for identification of quickly thinning upper clouds: 

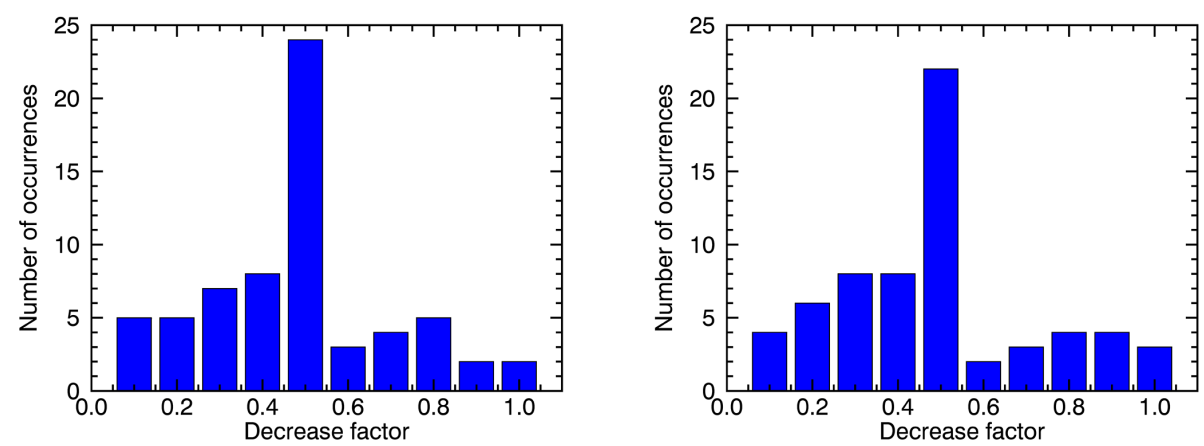

Figure 8. Distribution of the decrease factor $\Delta \tau / \Delta \tau_{\text {initial }}$ used to approximate the optical thickness decrease of a decaying cell (see text for details) for the 70 observed decaying cells for a forecast of $15 \mathrm{~min}$ (left) and $45 \mathrm{~min}$ (right).

- mean change in optical thickness from one time to the next is smaller than -0.01 ;

- mean divergence div (Eq. 3) of the motion vector field is smaller than -0.1 .

Thus, a decrease in optical thickness and a slight converging movement indicate a decaying cell.

To determine a typical correction term for the temporal evolution of upper cloud optical thickness after the decaying phase has started, the subset of all 70 decaying cells has been investigated closer. An empirical modification derived from them is imposed onto the optical thickness of the convective objects forecasted through the disparity vectors. Before the application of disparity vectors as described in Sect. 3.2.2, optical thickness $\tau(P)$ of each pixel $P$ inside the convective object is decreased by $f \cdot \Delta \tau . f$ is the number of time steps after the forecast starts and $\Delta \tau$ is an empirical average optical thickness step found using the mentioned 70 cases:

$\tau_{\text {corr }}(P)=\tau(P)+\Delta \tau \cdot f$.

On its turn, the typical step $\Delta \tau$ is parameterized as a function of the observed mean optical thickness decrease $\Delta \tau_{\text {initial }}$ of the convective object's optical thickness between the two initial images. This information, $\Delta \tau_{\text {initial }}$, is selected because it depends on the convective cell under observation and because it is representative to the given atmospheric and physical conditions encountered. For application within the forecast procedure, the occurrence of $\Delta \tau / \Delta \tau_{\text {initial }}$ in the range $[0,1]$ in bins of size 0.1 is investigated and shown in Fig. 8 for a forecast of $15 \mathrm{~min}$ (left) and $45 \mathrm{~min}$ (right), where $\Delta \tau$ is the mean observed cell optical thickness decrease. It turns out that the mean initial optical thickness decrease of the convective cell is the strongest one and that the most typical decrease corresponds to half this value for all forecast lead times up to $1 \mathrm{~h}$. Therefore, forecasts for all decaying cells are implemented using a decrease $\Delta \tau=0.5 \cdot \Delta \tau_{\text {initial }}$ in Eq. 4 . This method is not reasonable for a forecast of more than $1 \mathrm{~h}$ for the following reasons: (1) the remnants of the cells may merge with other clouds and are not detectable any more;
(2) the forecast and the observation of the cell differ strongly in shape, size and position. Thus, for a forecast of more than $1 \mathrm{~h}$ no further decrease in optical thickness is applied.

One example of a decaying cell is shown in Fig. 9. The object-based forecast for $30 \mathrm{~min}$ without intensity correction (left) predicts a larger ice optical thickness for the cell than it is in reality (middle). Figure 9 (right) depicts the effect of the intensity correction. The upper cloud optical thickness predicted by application of this intensity correction is lower and more realistic than for the original forecast (left).

\subsection{Step 4: synthesis}

After classification into upper and lower layer, object-based forecast of these layers and special correction for decaying upper layer convective cirrus clouds, two fields of possibly overlapping forecast optical thickness are available. Figure $3 \mathrm{~h}$ shows, comparable to Fig. $3 \mathrm{f}$, the $1 \mathrm{~h}$ forecast of upper cloud optical thickness and Fig. 3g the corresponding disparity vector field on top of the second initial image. By comparing both forecasts (Fig. 3f and Fig. 3h) the higher accuracy of the final forecast in terms of cloud coverage and optical thickness is clearly visible. In particular, the thin cloud patches in the northern part are better represented, the front is moving in a more compact way and the shape of cloudfree regions in the south is more realistic. However, the final forecast appears smoother than the observation because of the averaging procedure implemented for the cloud and cloud-free objects and because of the interpolation procedure implemented in Eq. (1).

\subsection{Step 5: calculation of DNI}

DNI computed in this paper considers only photons coming from the Sun that do not interact with the atmosphere (see the "strict definition" of DNI for numerical modeling of radiative transfer in Blanc et al., 2014). In particular, neither circumsolar radiation nor 3-D radiative effects are taken into 


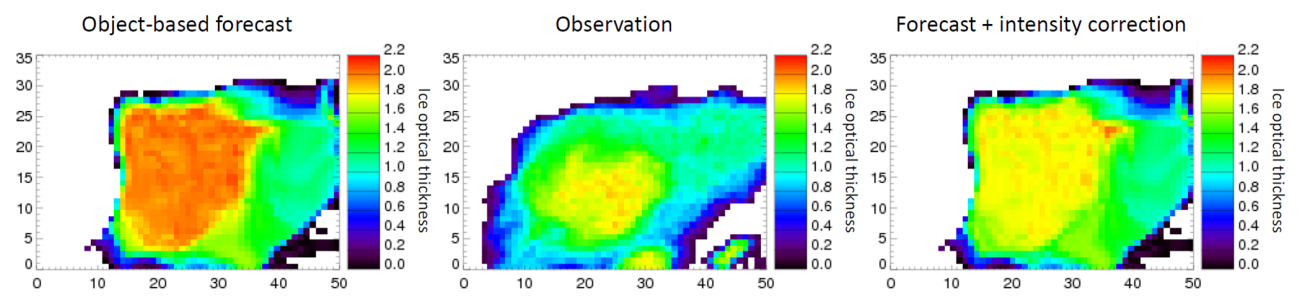

Figure 9. Upper cloud optical thickness for 9 June 2013, 17:00 UTC, for the real situation (middle) compared to the object-based forecast for $30 \mathrm{~min}$ (left) and the forecast with intensity correction (right).
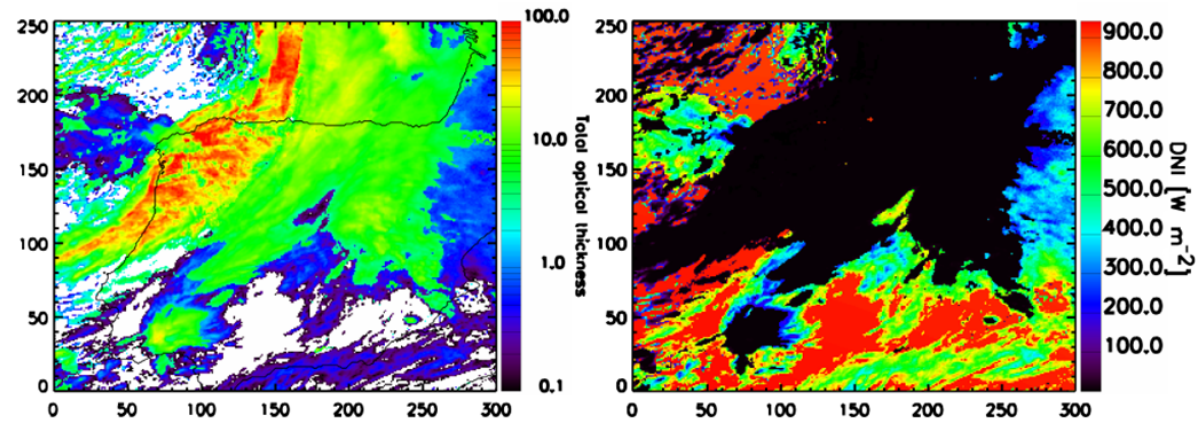

Figure 10. Optical thickness for upper and lower clouds together for 7 April 2013, 13:15 UTC (left), and the calculated direct normal irradiance in $\mathrm{W} \mathrm{m}^{-2}$ (right).

account. Thus,

$\mathrm{DNI}=\int E_{0}(\lambda) \cdot \exp \left(-\tau(\lambda) / \cos \left(\theta_{0}\right)\right) d \lambda$

according to the Lambert-Beer law. Here, the integral over wavelength $\lambda$ extends over the entire solar spectrum, $E_{0}(\lambda)$ represents the incoming solar radiation spectrum at top of atmosphere, $\theta_{0}$ the solar zenith angle and $\tau(\lambda)$ the vertical optical thickness of the atmosphere, including clouds, aerosols and (trace) gases. In the following, Eq. (5) is then approximated by means of

$\mathrm{DNI}=I_{0} \cdot \exp \left(-\left(\tau_{g}+\tau_{\mathrm{cld}}\right) / \cos \left(\theta_{0}\right)\right)$,

with the broadband extraterrestrial solar irradiance $I_{0}=$ $\int E_{0}(\lambda) d \lambda$. In Eq. (6), $\tau_{\text {cld }}$ represents the "broadband" cloud optical thickness and $\tau_{g}$ the "broadband" optical thickness of the atmosphere, which mainly depends on water vapor absorption and Rayleigh scattering (Gueymard, 2012). Since only cloud optical properties are derived from SEVIRI, aerosols are neglected while atmospheric transmission is mimicked with $\exp \left(-\tau_{g} / \cos \left(\theta_{0}\right)\right)$ and a typical value $\tau_{g}=0.292$ for a midlatitude summer standard atmosphere (Anderson et al., 1986). For the term $\tau_{\text {cld }}$, we use the cloud optical thickness at $550 \mathrm{~nm} \tau_{\text {cld, } 550 \mathrm{~nm}}$ that is output of the cloud retrievals APICS and COCS since the difference is very small $(<1 \%$ as revealed by radiative transfer computations).

Figure 10 depicts the total cloud optical thickness provided by adding up lower and upper layer's values (left) for the same scene as in Fig. 3 and the computed DNI (right). The values range from $0 \mathrm{~W} \mathrm{~m}^{-2}$ for areas with thick clouds (black) to around $900 \mathrm{~W} \mathrm{~m}^{-2}$ for cloud-free areas. Thin clouds reduce the DNI according to Eq. (6), as can be seen in the lower right corner.

\section{Validation}

For a validation of cloud and DNI forecasts two time periods, 4-31 March 2013 and 12-31 July 2013, were examined. These 2 months were chosen due to the appearance of different cloud types. The domain considered is the central part (marked in red in Fig. 6) of the area investigated in Sect. 3.3 with a size of $751 \times 501$ pixels. For March primarily advective clouds are present in this domain with an increasing amount of convective clouds in July. During daytime (solar zenith angle $<80^{\circ}$ ) a forecast is started each full hour with a forecast horizon of $2 \mathrm{~h}$ and a time step of $5 \mathrm{~min}$. Forecasts are compared to observations from SEVIRI. They represent the best result that a forecast algorithm based on such data can achieve. This is not an absolute validation of cloud cover and DNI, but it does take into account that the forecast can only be as good as the input quantities are.

\subsection{Cloud masks}

In order to quantitatively assess the performance of the forecast algorithm, we evaluate its capability to predict clouds and cloud-free pixels by examining the errors of the fore- 
Table 2. Contingency table.

\begin{tabular}{lllll}
\hline \multirow{2}{*}{ Forecast } & \multicolumn{4}{c}{ Observation } \\
\cline { 2 - 5 } & Scenario & Cloudy & Cloud-free & Total \\
\hline \multirow{2}{*}{ Cloudy } & $a$ & $b$ & $a+b$ \\
& Cloud-free & $c$ & $d$ & $c+d$ \\
\hline & Total & $a+c$ & $b+d$ & $N=a+b+c+d$ \\
\hline
\end{tabular}

cast cloud mask against observed cloud masks from SEVIRI. Observations and forecast are connected through the contingency table (Table 2). Its four elements are the hits $a$, misses $c$, false alarms $b$ and correct negative events $d$. Hits represent the number of pixels that are correctly forecasted as cloudy. Misses are the number of pixels that have been falsely predicted as cloud free although the observation is cloudy. False alarms are the number of pixels that are falsely predicted as cloudy although observations classify them as cloud free. Correct negatives are the number of pixels that are correctly forecasted as cloud free. Figure 11 shows the four elements (hits in red, false alarms (fa) in green, misses in blue and correct negatives (cn) in white) for upper (left) and lower (right) cloud layers. Errors, especially for lower clouds, mainly occur at cloud edges or due to new developments or dissipating clouds.

The calculated parameters of the contingency table for all start times are averaged for every forecast time step up to $2 \mathrm{~h}$ (see beginning of Sect. 4 for the illustration of the forecast data set evaluated here). In Fig. 12 (left) the forecast errors (misses plus false alarms) for March (triangles) and July (crosses) are shown in percent with respect to the total number of pixels in the scene for upper (red) and lower (blue) clouds. For the upper cloud layer (but not for the lower clouds, see below) errors for persistence are plotted for comparison. This comparison illustrates the benefit of the developed forecast algorithm. Forecasts errors are significantly lower compared to persistence with the smallest values for the $5 \mathrm{~min}$ forecasts (errors below $5 \%$ for upper cloud layer). At this time, persistence is still close to the observation, because clouds change only slightly during this time step. Afterwards, forecast errors increase smoothly with every time step to a maximum after $2 \mathrm{~h}$. The difference between persistence and forecast increases also with time. For instance, for the upper cloud layer errors reached by persistence after about $1 \mathrm{~h}$ are reached by the forecast only after $2 \mathrm{~h}$ for March and July. Compared to persistence, the forecast at least doubles the lead time at a certain quality level. Most noticeable are the differences between the two cloud layers. The performance of the algorithm for the upper clouds shows much better results than for lower clouds. The reasons are

1. the difficulty of a retrieval for lower clouds below thick upper clouds, which leads to errors in the forecast when not all clouds are detected in the initial images, a low cloud layer disappears below a high one or low clouds evolve into high clouds;

2. larger small-scale variability for lower clouds, which cannot be resolved by SEVIRI (Wolters et al., 2010; Koren et al., 2008);

3. sub-pixel inhomogeneity, i.e., broken cumulus cloud fields and rapidly changing small-scale convective cloud fields with very short timescales but low advection speeds (Bley et al., 2016);

4. formation of new lower clouds that cannot be forecasted.

Errors are smaller in July compared to March because of the low cloud cover of $22.1 \%$ on average $(62.7 \%$ in March) during this month (errors are relative to satellite scene size). For water clouds, detection and forecast are hindered by the presence of upper clouds such that even a correct forecast might be incorrectly classified. Thus, it is difficult to assess the real accuracy of water cloud forecasts. For this reason, persistence for water clouds has not been evaluated.

In addition to the evaluation of the errors as shown above, we apply the Hanssen-Kuiper skill score (HK; Hanssen and Kuipers, 1965) to our data set. This score has been widely used for the evaluation of meteorological fields for many years (Woodcock, 1976). It has been applied in particular to precipitation forecasts (e.g., Doswell et al., 1990; Stephenson, 2000; Accadia et al., 2003; Tartaglione, 2010; Gsella et al., 2014; Fekri and Yau, 2016) against observations but also cloud retrieval algorithms (Reuter et al., 2009; Bugliaro et al., 2011; Reuter and Fischer, 2014).

The HK, also called Hanssen-Kuiper discriminant, Peirce skill score (Peirce, 1884), or true skill score (Flueck, 1987), combines the four elements of the contingency table (Table 2) in the following way:

$$
\begin{aligned}
\mathrm{HK} & =\frac{a d-b c}{(a+c)(b+d)} \\
& =\frac{a}{a+c}+\frac{d}{b+d}-1 \\
& =\frac{a}{a+c}-\frac{b}{b+d} .
\end{aligned}
$$

It can be expressed as the sum of the accuracy for events, i.e., the accuracy of forecasted clouds (first term in Eq. 7, $\frac{a}{a+c}$, also called hit rate, $H$, or probability of detection, POD), and the accuracy for non-events, i.e., the accuracy of forecasted cloud-free pixels (second term in Eq. $7, \frac{d}{b+d}$ ). The subtraction of 1 in the end ensures that $-1<\mathrm{HK}<1$. The HK can also be expressed as the difference between the hit rate $H=\frac{a}{a+c}$ (first term in Eq. 8) and the false alarm rate, $F=\frac{b}{b+d}$, or probability of false detection (PODF; second term in Eq. 8). Thus, HK is a measure of the hit rate relative to the false alarm rate and remains positive as long as 

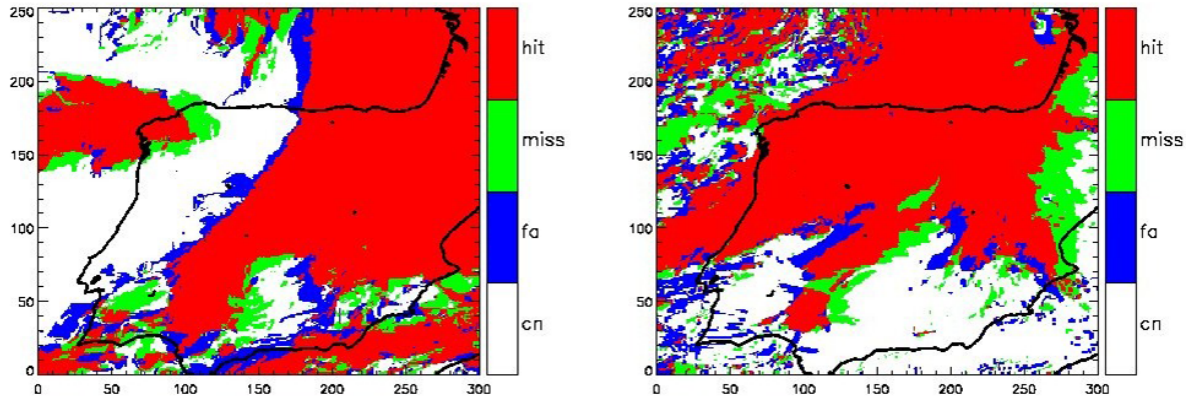

Figure 11. Illustration of the elements of the contingency table for upper (left) and lower (right) clouds with regard to the $1 \mathrm{~h}$ forecast for 7 April 2013, 14:15 UTC: hits in red, false alarms (fa) in blue, misses in green and correct negatives (cn) in white.
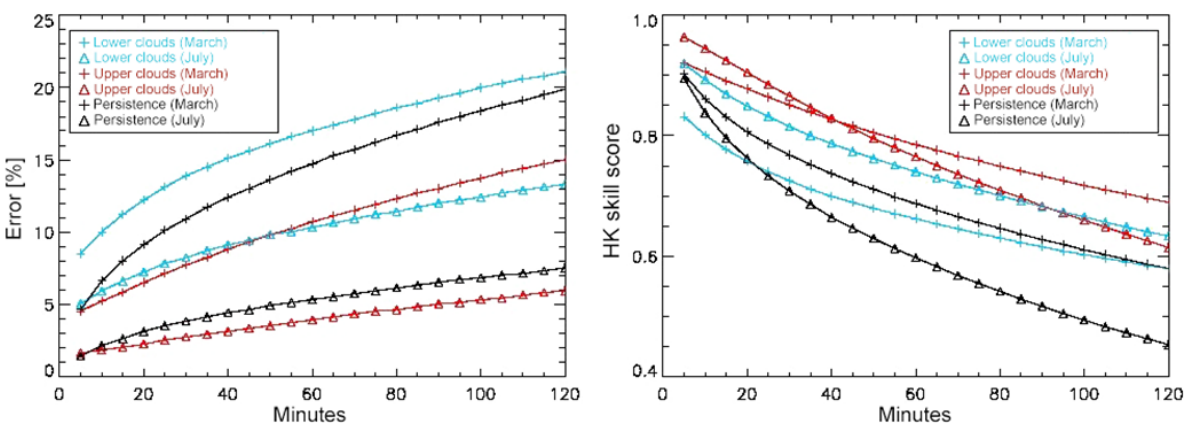

Figure 12. (a) Forecast errors (misses plus false alarms) and (b) the Hanssen-Kuiper skill score for March (triangles) and July (crosses) in percent for upper (red) and lower (blue) cloud layers and persistence for upper clouds (black).

$H$ is larger than $F$, i.e., indicates the ability of the forecast algorithm to produce correct cloud forecasts as well as to avoid false alarms. A skill score of 1 denotes a perfect match (all detected clouds have been forecasted, misses $c$ and false alarms $b$ are zero), a score equal to -1 is related to a forecast not matching at all (hits $a$ and correct negatives $d$ are zero). Negative values are related to "inverse" forecasts and could be turned into positive values by interchanging forecasted events and non-events. A score of 0 is produced, e.g., by a forecast of a fully cloudy or fully cloud-free scene, or by a "random" forecast, i.e., when $H$ and $F$ are equal. In this sense, the HK represents the accuracy of the forecast in predicting the correct category with respect to the ability of a random selection. Furthermore, HK is independent of the relative frequency of the observations and also works with asymmetrical distributions; i.e., when more cloudy than cloud-free pixels are present or vice versa. This is an important feature of this skill score since different geographical areas, different seasons and different times of the day are characterized by different cloud amounts that can vary considerably. In fact, if the cloud cover is low, i.e., if clear-sky cover $(b+d)$ is large $(a+c \ll b+d)$, it is easy to correctly forecast $(d)$ the largest part of it, as errors can only arise from small edge areas of small cloud cover (the forecast procedure does not account for cloud formation but rather shifts and modifies the shape of existing clouds). Thus, the sec- ond term in Eq. (7) is large, i.e., the second term in Eq. (8) is small (few false alarms). This tendency towards large HK contributions due to high non-event accuracy (high accuracy of cloud-free pixels) is balanced by the first term of the HK both in Eqs. (7) and (8). There the same error potential (misplaced cloud edges) leads to large cloud errors $(a)$ compared to the small cloud cover $(a+b)$. A low score contribution from events, i.e., a low hit rate, is the result. This way the direct effect of cloud cover on the skill score is minimized.

The resulting HK (Fig. 12, right) has been determined for upper (red) and lower (blue) clouds for both months with high values above 0.9 for the first time steps except for lower clouds in March (0.8) and a decrease to $0.55-0.7$ after $2 \mathrm{~h}$. As shown before the forecasts for upper clouds perform better than for lower clouds. The HK for persistence for the upper clouds (black lines) is significantly lower than the respective forecasts (red lines) especially for July (black triangles vs. red triangles). Here, persistence already shows a lower skill for a $5 \mathrm{~min}$ forecast. This is mainly due to the lower hit rate of the persistence method with respect to the forecast in a situation where few clouds are present in the area under study (July has low cloud cover, see above): already a small displacement of the clouds can lead to significantly lower hit rates $H$ in this situation (see discussion of the HK presented above). Differences of the accuracy of persistence for upper clouds between March and July are evident: the two black 

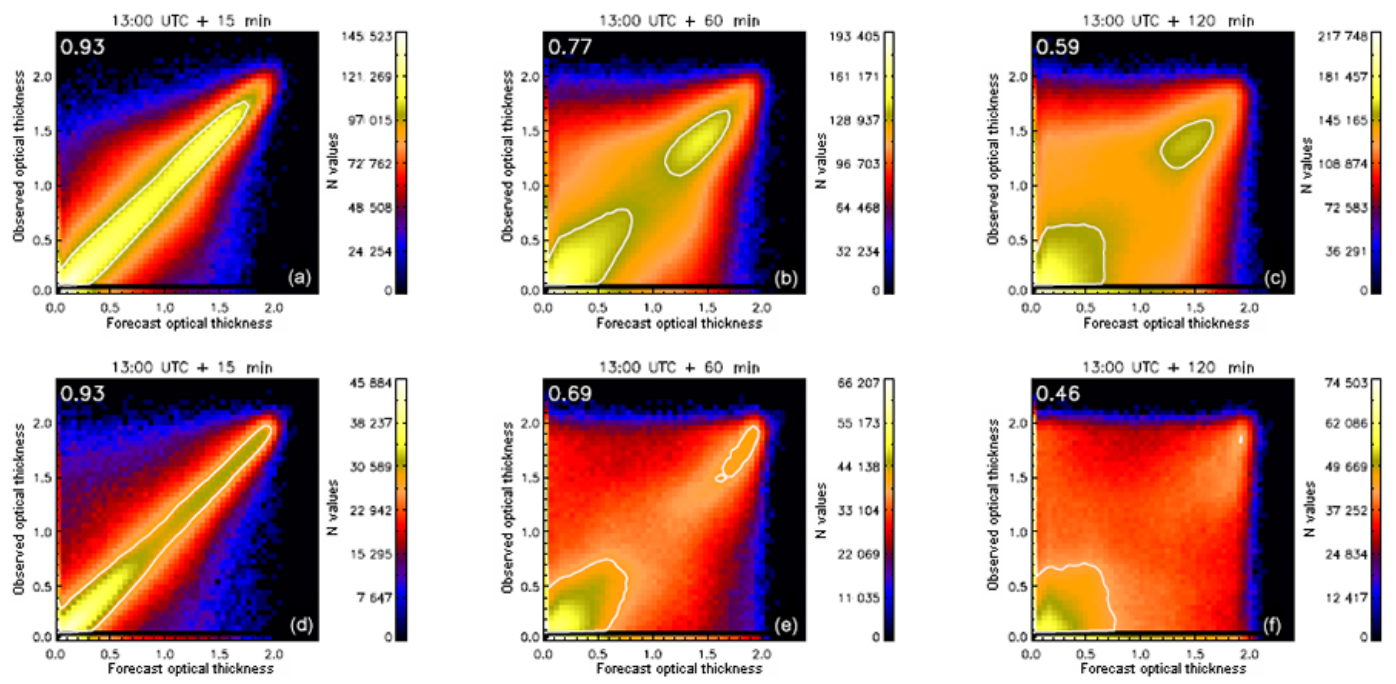

Figure 13. Two-dimensional histogram of the forecasted optical thickness of the upper cloud layer compared to the real optical thickness with forecasts starting at 13:00 UTC every day: for a $15 \mathrm{~min}$ forecast (a) $1 \mathrm{~h}$ forecast (b) and $2 \mathrm{~h}$ forecast (c) in March and July respectively $(\mathbf{d}, \mathbf{e}, \mathbf{f})$. Colors denote the total number of occurrences. The number in the upper left corner of all images is the correlation coefficient. The white contour line denotes the $90 \%$ percentile.
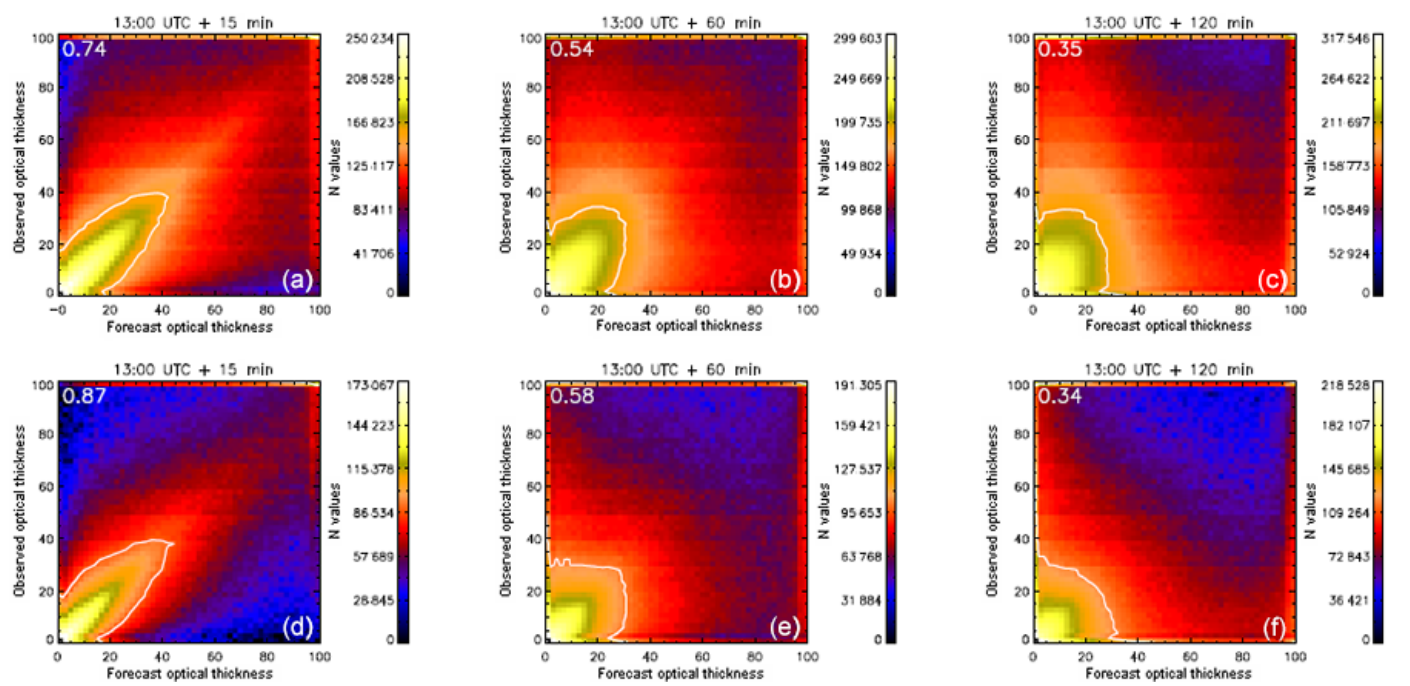

Figure 14. Two-dimensional histogram of the forecasted optical thickness for the lower cloud layer compared to the real optical thickness with forecasts starting at 13:00 UTC every day: for a $15 \mathrm{~min}$ forecast (a), $1 \mathrm{~h}$ forecast (b) and $2 \mathrm{~h}$ forecast (c) in March and July respectively $(\mathbf{d}, \mathbf{e}, \mathbf{f})$. Colors denote the total number of occurrences. The number in the upper left corner of all images is the correlation coefficient. The white contour line denotes the $90 \%$ percentile.

lines in Fig. 12 (right) diverge with time, a hint that upper cloud forecasts for July are more difficult than for March due to the predominantly convective nature of clouds in July and to the inability of the matcher to forecast convective initiation and phase transition from liquid to solid during the convective process. However, the red curves in Fig. 12 (right) show that the HK of the upper cloud forecast becomes lower for July than for March for lead times larger than $40 \mathrm{~min}$. The faster score decrease in July is due to the newly developing convective clouds for which the forecast becomes inaccurate within a short period of time. The resulting larger cloud errors lead to lower values for July in the first term in Eq. (7) while the second term shows constantly high values due to the low cloud cover. The higher values in July for the first $40 \mathrm{~min}$ in the HK originate from the score contributions due to high cloud-free pixels (non-events) accuracy (first term in Eq. 7). Nevertheless, the benefit in skill of the forecast compared to persistence can again be expressed as more than a doubling of lead times for a given score level. For the lower cloud layer (blue curves in Fig. 12, right) the performance is 
better in July (blue triangles) than in March (blue crosses). This arises from the combination of (1) a higher hit rate in March than in July, due to the larger lower cloud extent in March than in July and to the usually larger lower cloud sizes in March than in July (think of the frequent appearance of scattered cumuli in July) that makes it easier to forecast lower clouds in March, and (2) a higher false alarm rate in March than in July mainly due to the higher upper cloud coverage in March and the associated lower detection accuracy of lower clouds. The second effect outweighs the first one such that lower cloud forecasts in July are more accurate than in March.

\subsection{Cloud optical thickness}

In order to test the performance of the algorithm with regard to the optical thickness a comparison of the forecasted optical thickness with the optical thickness observed from SEVIRI is done via a 2-D histogram separated into upper (Fig. 13) and lower cloud layers (Fig. 14). The color bar denotes the total number of occurrences. We select the forecast starting at 13:00 UTC on each day in March (Figs. 13 and 14a, b, c) and July (Figs. 13 and 14d, e, f) and compare forecast steps with the actual SEVIRI data measured. Comparisons of optical thickness for a $15 \mathrm{~min}, 1 \mathrm{~h}$ and $2 \mathrm{~h}$ forecast with observed optical thickness are shown separately in Fig. 13 for the higher and in Fig. 14 for the lower cloud layer.

For the upper cloud layer the algorithm shows an overall good performance with only small differences for most of the pixels for the 15 min forecast (Fig. 13a, d) and an increase of spread for the $1 \mathrm{~h}$ forecast (Fig. 13b, e) and $2 \mathrm{~h}$ forecast (Fig. 13c, f), which is illustrated by the $90 \%$ percentile (white contour line). The fact that the COCS algorithm produces results of either 0 or the range $[0.1,2.5]$ creates the narrow line without values between 0 and 0.1 . Larger deviations mainly occur for observed or forecasted optical thickness equal to zero, where clouds have been forecasted but not observed and vice versa (false alarms and misses). Remarkable is the existence of two maxima in good agreement for small optical thickness around 0.25 and large values around 1.5. These reflect the two types of high clouds mostly occurring: thin cirrus clouds and optical thick upper parts of deep clouds. The corresponding correlation coefficients $(>0.9)$ are also shown and they confirm the good performance of the algorithm for the upper clouds for the first forecast time steps. In analogy to the analysis of the HK, the correlation coefficients show higher values in March than in July, in particular for later time steps. This is most likely a consequence of the high frequency of convective clouds in July.

For the lower cloud layer (Fig. 14) the distribution is broader due to the mentioned limitations of detection of lower clouds below higher ones. This is particularly true for March (Fig. 14a, b, c) and obvious in the mismatch for observed optical thickness at the largest values of optical thick- ness. This seems to be more difficult for March than for July, most likely because of multilayered clouds around frontal systems.

The corresponding correlation coefficients show high values above 0.74 for the first time steps despite the lack in skill for cloud detection of lower clouds. Its deteriorating influence is apparent in the sharp decrease of the correlation coefficients for the 1 and $2 \mathrm{~h}$ forecast.

To judge the quality of the forecast algorithm, histograms of the persistence method for the upper cloud layer are shown in Fig. 15. Compared to forecasts in Figure 13, deviation distributions are much broader and correlation coefficients significantly lower.

\subsection{Direct normal irradiance}

Figure 16 illustrates the comparison of forecasted and observed DNI (Sect. 3.5) analogous to Figs. 13 and 14. Two maxima can be observed: (1) for low DNI in case of thick clouds and (2) for high DNI around $800 \mathrm{~W} \mathrm{~m}^{-2}$ for March (Fig. 16a, b, c) and $900 \mathrm{~W} \mathrm{~m}^{-2}$ for July (Fig. 16d, e, f) for cloud-free cases with varying solar zenith angle. In analogy to the comparison of optical thickness, high deviations arise from cloud cover false alarms and misses and because of the difficulties of detecting multilayer clouds. Remarkable is the sharply defined region at high DNI values $\left(>600 \mathrm{~W} \mathrm{~m}^{-2}\right)$ showing a clear deficit of cases close to the $1: 1$ line. This is due to the fact that COCS does not provide measured values of optical thickness below 0.1. Thus large DNI values are missing in the "observation", while the forecast can produce these optical thickness and DNI values.

The correlation coefficients for DNI are mostly higher than the values of both cloud types, especially for long forecasts, with lower values in July. This is due to the fact that forecasts are better for cloud areas with small optical thickness values than for optically thick clouds. Derivation of DNI emphasizes the relevance of these thin clouds for DNI predictions, while errors in the forecast of thick opaque clouds (e.g., new convective developments) are less detrimental.

\section{Conclusions}

Based on an optical flow method deriving cloud motion between two consecutive images, an algorithm for the forecast of cloud optical thickness and DNI has been developed for input data from the imager SEVIRI aboard the geostationary MSG satellite. The algorithms COCS and APICS provide cloud detection and cloud optical thickness for two vertically separated layers. Because of different velocities and motion directions these low and high level clouds are forecasted separately for a time step of 5 min with a forecast horizon up to $2 \mathrm{~h}$. To deal with the small-scale fluctuations of the motion field derived for the two levels, which would spoil forecasts of more than 15 or $30 \mathrm{~min}$, an object classification is applied 

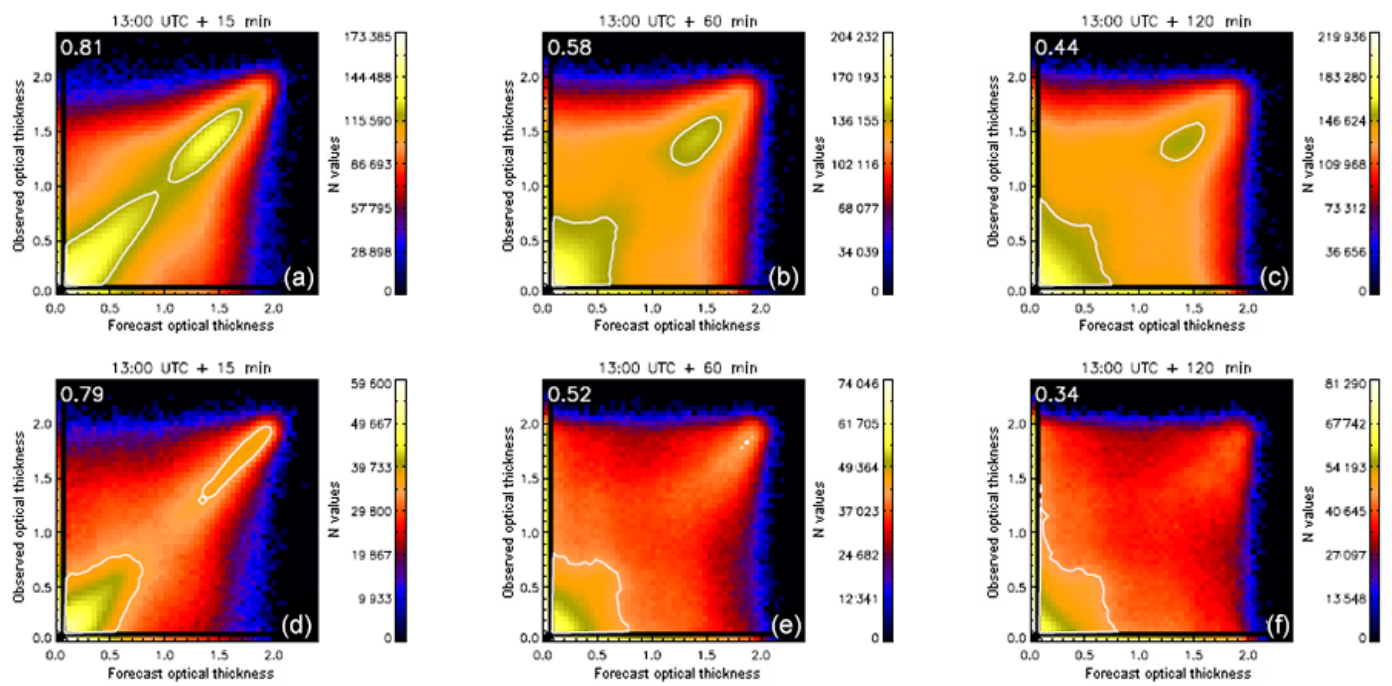

Figure 15. Two-dimensional histogram of the observed optical thickness of the upper cloud layer compared to the persistence optical thickness for a time difference of $15 \mathrm{~min}(\mathbf{a}), 1 \mathrm{~h}$ (b) and $2 \mathrm{~h}$ (c) in March and July respectively (d, e, f). Colors denote the total number of occurrences. The white contour line denotes the $90 \%$ percentile.
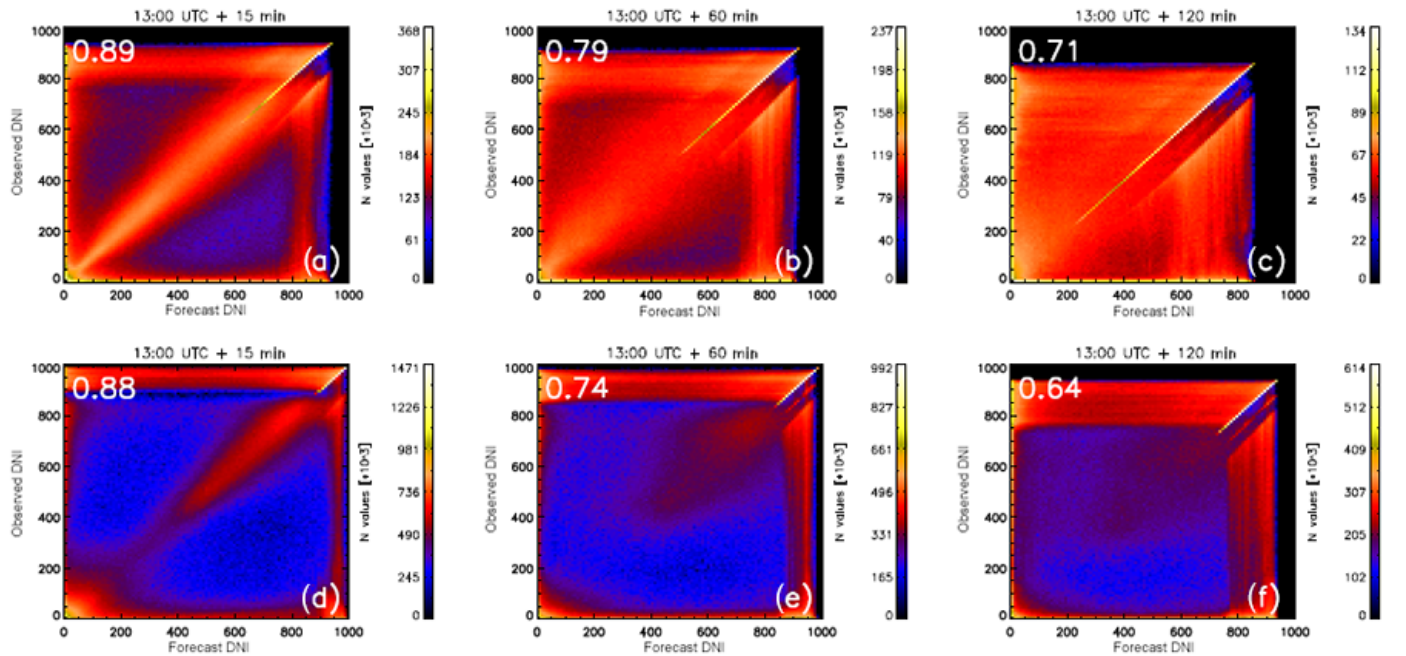

Figure 16. Two-dimensional histogram of the forecasted DNI compared to the observed DNI with forecasts starting at 13:00 UTC every day: for a $15 \mathrm{~min}$ forecast (a), $1 \mathrm{~h}$ forecast (b) and $2 \mathrm{~h}$ forecast (c) in March and July respectively (d, e, f). Colors denote the total number of occurrences.

to the cloud layers and cloud-free background motion is interpolated. An intensity correction for decaying convective cells is implemented.

Using the satellite observations, the forecasts for March and July 2013 have been quantitatively validated. As far as cloud detection is concerned, the largest inaccuracy consists in the difficulty to retrieve clouds below optically thick ice clouds. Consequently, forecast errors for the lower cloud layer are considerably higher than for high clouds. The forecast accuracy also differs for the two time periods because of different cloud coverage and cloud types. In March mainly fronts with many advective multilayer clouds dominate in contrast to a high amount of shorter-lived convective clouds in July.

Convective clouds during July cause the forecast skill to decay quicker with forecast horizon than during March. For any given forecast quality requirement, over all cloud (or weather) types and for both cloud layers, a doubling of lead time is found comparing the developed forecast to a nonforecast, i.e., persistence.

The impact of weather situation also becomes apparent in the comparison of observed and forecasted optical thickness. The distribution of deviations, analyzed by means of 2-D histograms, as well as the correlation between forecast and 
observation show better results for March, especially for a longer forecast. The wider scatter of deviations as well as lower correlation coefficients confirm the limitations of the forecast quality for low clouds compared to high clouds. Although much effort was invested in the identification of multilayer clouds and their differential motion, this still remains a main source of uncertainty for satellite-based nowcasting. An additional source of uncertainty is sub-pixel inhomogeneity, i.e., broken cumulus cloud fields and rapidly changing small-scale convective cloud fields with very short timescales but low advection speeds (Bley et al., 2016).

Finally, DNI forecast verification shows that most correct forecasts are, of course, found for the expected clear-sky DNI and no direct irradiance below thick clouds. However, the overall correlation between the $2 \mathrm{~h}$ forecast and the observation is still around 0.7.

As a next step comparisons to ground-based irradiance measurements shall be conducted. To this end, the DNI model should be extended to consider varying trace gas concentrations and aerosol loads, e.g., water vapor from numerical weather models and aerosol information from groundbased networks (e.g., AERONET).

An extension of the forecast horizon up to 5-6 h could be performed, which is indeed useful for CSP operators. For longer forecast horizons forecasting methods of NWP models have a higher accuracy compared to satellite-based methods (Perez et al., 2013; Lorenz et al., 2012) and are therefore used by operators.

\section{Data availability}

The MSG/SEVIRI level 1.5 data are available at http://www.eumetsat.int/website/home/Data/DataDelivery/ OnlineDataAccess/index.html. The processed data are available from the author upon request.

Competing interests. The authors declare that they have no conflict of interest.

Acknowledgements. We acknowledge the European Commission for funding the project DNICast (www.dnicast-project.net), grant agreement 608623, and our colleague Hermann Mannstein, who passed away much too early in 2011 , for a wealth of inventive and clever ideas that led to these results and for the development of the cloud matcher.

The article processing charges for this open-access publication were covered by a Research

Centre of the Helmholtz Association.

Edited by: A. Kokhanovsky

Reviewed by: three anonymous referees

\section{References}

Accadia, C., Mariani, S., Casaioli, M., Lavagnini, A., and Speranza, A.: Sensitivity of Precipitation Forecast Skill Scores to Bilinear Interpolation and a Simple NearestNeighbor Average Method on High-Resolution Verification Grids, Weather Forecast., 18, 918-932, doi:10.1175/15200434(2003)018<0918:SOPFSS>2.0.CO;2, 2003.

Anderson, G., Clough, S., Kneizys, F., Chetwynd, J., and Shettle, E.: AFGL Atmospheric Constituent Profiles (0-120 km), Tech. Rep. AFGL-TR-86-0110, AFGL (OPI), Hanscom AFB, MA 01736, 1986.

Baum, B., Uttal, T., Poellot, M., Ackermann, T., Alvarez, J., Intrieri, J., Starr, D., Titlow, J., Tovinkere, V., and Clothiaux, E.: Satellite remote sensing of multiple cloud layers, J. Atmos. Sci., 52, 4210-4230, 1995.

Baum, B., Heymsfield, A., Yang, P., and Bedka, S.: Bulk scattering models for the remote sensing of ice clouds. Part 1: Microphysical data and models, J. Appl. Meteor., 44, 1885-1895, doi:10.1175/JAM2308.1, 2005.

Blanc, P., Espinar, B., Geuder, N., Gueymard, C., Meyer, R., PitzPaal, R., Reinhardt, B., Renne, D., Sengupta, M., Wald, L., and Wilbert, S.: Direct normal irradiance related definitions and applications: The circumsolar issue, Sol. Energy, 110, 561-577, 2014.

Bley, S., Deneke, H., and Senf, F.: Meteosat-Based Characterization of the Spatio-Temporal Evolution of Warm Convective Cloud Fields over Central Europe, J. Appl. Meteor. Climatol., 55, 21812195, doi:10.1175/JAMC-D-15-0335.1, 2016.

Bolliger, M., Binder, P., and Rossa, A.: Tracking cloud patterns by METEOSAT rapid scan imagery in complex terrain, Meteorol. Z., 12, 73-80, 2003.

Bosch, J. and Kleissl, J.: Cloud motion vectors from a network of ground sensors in a solar power plant, Sol. Energy, 95, 13-20, doi:10.1016/j.solener.2013.05.027, 2013.

Bugliaro, L., Zinner, T., Keil, C., Mayer, B., Hollmann, R., Reuter, M., and Thomas, W.: Validation of cloud property retrievals with simulated satellite radiances: a case study for SEVIRI, Atmos. Chem. Phys., 11, 5603-5624, doi:10.5194/acp-11-5603-2011, 2011.

Byers, H. and Braham, R.: Thunderstorm Structure and Circulation, J. Meteorol., 5, 71-86, 1948.

Chu, Y., Pedro, H., and Coimbra, C.: Hybrid intra-hour DNI forecasts with sky image processing enhanced by stochastic learning, Sol. Energy, 98, 592-603, doi:10.1016/j.solener.2013.10.020, 2013.

Doswell, C. A., Davies-Jones, R., and Keller, D.: On Summary Measures of Skill in Rare Event Forecasting Based on Contingency Tables, Weather Forecast., 5, 576-585, doi:10.1175/15200434(1990)005<0576:OSMOSI>2.0.CO;2, 1990.

Eissa, Y., Marpu, P., Gherboudj, I., Ghedira, H., Ouarda, T., and Chiesa, M.: Artificial neural network based model for retrieval of the direct normal, diffuse horizontal and global horizontal irradiances using SEVIRI images, Sol. Energy, 89, 1-16, 2013.

Emde, C., Buras-Schnell, R., Kylling, A., Mayer, B., Gasteiger, J., Hamann, U., Kylling, J., Richter, B., Pause, C., Dowling, T., and Bugliaro, L.: The libRadtran software package for radiative transfer calculations (version 2.0.1), Geosci. Model Dev., 9, 1647-1672, doi:10.5194/gmd-9-1647-2016, 2016. 
Evans, A.: Cloud motion analysis using multichannel correlationrelaxation labeling, IEEE Geosci. Remote Sens. Lett., 3, 392396, 2006.

Feidas, H. and Cartarlis, C.: Application of an automated cloudtracking algorithm on satellite imagery for tracking and monitoring small mesoscale convective cloud systems, Int. J. Remote Sens., 26, 1677-1698, 2005.

Fekri, M. and Yau, M. K.: An Information-Theoretical Score of Dichotomous Precipitation Forecast, Mon. Weather Rev., 144, 1633-1647, doi:10.1175/MWR-D-15-0225.1, 2016.

Flueck, J. A.: A study of some measures of forecast verification, in: 10th Conference on Probability and Statistics in Atmospheric Sciences, 69-73, Amer. Meteor. Soc., Edmonton, AB, Canada, 1987.

Gonzalez, A., Mayer, B., Gayet, J., and Wendling, P.: Multilayer cirrus cloud property retrieval using dual-view ATSR-2 instrument, Proc. SPIE, 4882, 224-231, 2003.

Gonzalez, J., Serrano, E., and Wiesenberg, R.: DNI forecasting using coupled WRF and MRM, enhanced with a neural network for CSP applications, in: Proceedings of SolarPACES Conference, Perpignan, France, 2010.

Gsella, A., de Meij, A., Kerschbaumer, A., Reimer, E., Thunis, P., and Cuvelier, C.: Evaluation of MM5, \{WRF\} and \{TRAMPER\} meteorology over the complex terrain of the Po Valley, Italy, Atmos. Environ., 89, 797-806, doi:10.1016/j.atmosenv.2014.03.019, 2014.

Gueymard, C.: Clear-sky irradiance predictions for solar resource mapping and large-scale applications: Improved validation methodology and detailed performance analysis of 18 broadband radiative models, Sol. Energy, 86, 2145-2169, doi:10.1016/j.solener.2011.11.011, 2012.

Hanssen, A. W. and Kuipers, W.: On the relationship between the frequency of rain and various meteorological parameters, Koninklijk Ned. Meteor. Instit., Meded. Verhand., 2-15, 1965.

Henken, C. and Schmeits, M.: Using MSG-SEVIRI Cloud Physical Properties and weather Radar Observations for the Detection of Cb/TCu Clouds, J. Appl. Meteor. Clim., 50, 1587-1600, 2011.

Hering, A., Morel, C., Galli, G., Senesi, S., Ambrosetti, P., and Boscacci, M.: Nowcasting thunderstorms in the Alpine Region using a radar based adaptive thresholding scheme, in: Proc. ERAD Conference 2004, Visby, Sweden, 206-211, 2004.

Huang, J., Minnis, P., Lin, B., Yi, Y., Khaiyer, M., Arduini, R., Fan, A., and Mace, G.: Advanced retrievals of multilayered cloud properties using multispectral measurements, J. Geophys. Res., 110, doi:10.1029/2004JD005101, 2005.

Joiner, J., Vasilkov, A. P., Bhartia, P. K., Wind, G., Platnick, S., and Menzel, W. P.: Detection of multi-layer and vertically-extended clouds using A-train sensors, Atmos. Meas. Tech., 3, 233-247, doi:10.5194/amt-3-233-2010, 2010.

Koren, I., Oreopoulos, L., Feingold, G., Remer, L., and Altaratz, O.: How small is a small cloud?, Atmos. Chem. Phys., 8, 3855-3864, doi:10.5194/acp-8-3855-2008, 2008.

Kox, S., Bugliaro, L., and Ostler, A.: Retrieval of cirrus cloud optical thickness and top altitude from geostationary remote sensing, Atmos. Meas. Tech., 7, 3233-3246, doi:10.5194/amt-7-32332014, 2014.

Kraas, B., Schroedter-Homscheidt, M., and Madlener, R.: Economic merits of a state-of-the-art concentrating solar power fore- casting system for participation in the Spanish electricity market, Sol. Energy, 93, 244-255, 2013.

Kriebel, K.-T., Gesell, G., Kästner, M., and Mannstein, H.: The cloud analysis tool APOLLO: improvements and validation, Int. J. Remote Sens., 24, 2389-2408, doi:10.1080/01431160210163065, 2003.

Lang, P.: Cell Tracking and warning Indicators derived from Operational Radar Products, in: 30th International Conference on Radar Meteorology, Munich, Germany, 2001.

Lee, D.-T. and Schachter, B. J.: Two algorithms for constructing a Delaunay triangulation, Int. J. Comp. Inf. Sci., 9, 219-242, 1980.

Lorenz, E., Kühnert, J., and Heinemann, D.: Short term forecasting of solar irradiancs by combining satellite data and numerical weather predictions, in: 27th European Photovoltaic Sol. Energy Conference and Exhibition, Frankfurt 4401-4405, 2012.

Marquez, R. and Coimbra, C.: Forecasting of global direct solar irradiance using stochastic learning methods, ground experiments and the $\{$ NWS $\}$ database, Sol. Energy, 85, 746-756, 2011.

Marquez, R. and Coimbra, C.: Intra-hour $\{\mathrm{DNI}\}$ forecasting based on cloud tracking image analysis, Sol. Energy, 91, 327-336, 2013.

Mayer, B. and Kylling, A.: Technical Note: The libRadtran software package for radiative transfer calculations: Description and examples of use, Atmos. Chem. Phys., 5, 1855-1877, doi:10.5194/acp-5-1855-2005, 2005.

Merk, D. and Zinner, T.: Detection of convective initiation using Meteosat SEVIRI: implementation in and verification with the tracking and nowcasting algorithm Cb-TRAM, Atmos. Meas. Tech., 6, 1903-1918, doi:10.5194/amt-6-1903-2013, 2013.

Nakajima, T. and King, M.: Determination of the optical thickness and effective particle radius of clouds from reflected solar radiation measurements. Part I: Theory, J. Atmos. Sci., 47, 1878-1893, doi:10.1175/15200469(1990)047<1878:DOTOTA>2.0.CO;2, 1990.

Nakajima, T. and Nakajima, T.: Wide-area determination of cloud microphysical properties from NOAA AVHRR measurements for FIRE and ASTEX regions, J. Atmos. Sci., 52, 4043-4059, 1995.

Peirce, C. S.: The numerical measure of the success of predictions, Science, 4, 453-454, 1884.

Perez, R., Kivalov, S., Schlemmer, J., Hemker Jr., K., Renne, D., and Hoff, T.: Validation of short and medium term operational solar radiation forecasts in the US, Sol. Energy, 84, 2161-2172, 2013.

Pierce, C., Hardaker, P., Collier, C., and Haggett, C.: GANDOLF: a system for generating automated nowcasts of convective precipitation, Meteorol. Appl., 7, 341-360, 2000.

Reuter, M. and Fischer, J.: A comparison of satellite-retrieved and simulated cloud coverage in the Baltic Sea area as part of the BALTIMOS project, Theor. Appl. Climatol., 118, 695-706, doi:10.1007/s00704-009-0208-8, 2014.

Reuter, M., Thomas, W., Albert, P., Lockhoff, M., Weber, R., Karlsson, K.-G., and Fischer, J.: The CM-SAF and FUB Cloud Detection Schemes for SEVIRI: Validation with Synoptic Data and Initial Comparison with MODIS and CALIPSO, J. Appl. Meteor. Climatol., 48, 301-316, doi:10.1175/2008JAMC1982.1, 2009.

Schaaf, C., Gao, F., Strahler, A., Lucht, W., Li, X., Tsang, T., Strugnell, N., Zhang, X., Jin, Y., Muller, J.-P., Lewis, P., Barnsley, M., Hobson, P., Disney, M., Roberts, G., Dunderdale, M., 
Doll, C., d'Entremont, R., Hu, B., Liang, S., and Privette, J.: First Operational BRDF, Albedo and Nadir Reflectance Products from MODIS, Remote Sens. Envir., 83, 135-148, 2002.

Schmetz, J., Holmlund, K., Hoffman, J., Strauss, B., Mason, B., Gaertner, V., Koch, A., and Van De Berg, L.: Operational cloudmotion winds from Meteosat infrared images, J. Appl. Meteorol., 32, 1206-1225, 1993a.

Schmetz, J., Holmlund, K., Hoffman, J., Strauss, B., Mason, B., Gaertner, V., Koch, A., and van de Berg, L.: Operational cloudmotion winds from Meteosat infrared images, J. Appl. Meteor., 32, 1206-1225, 1993b.

Schmetz, J., Pili, P., Tjemkes, S., Just, D., Kerkmann, J., Rota, S., and Ratier, A.: An introduction to Meteosat Second Generation (MSG), B. Am. Meteor. Soc., 83, 977-992, doi:10.1175/15200477(2002)083<0977:AITMSG>2.3.CO;2, 2002.

Shettle, E.: Models of aerosols, clouds and precipitation for atmospheric propagation studies, in: Atmospheric propagation in the uv, visible, ir and mm-region and related system aspects, no. 454 in AGARD Conference Proceedings, 1989.

Steinacker, R., Dorninger, M., Wölfelmaier, F., and Krennert, T.: Automatic Tracking of convective cells and cell complexes from lightning and radar data, Meteorol. Atmos. Phys., 72, 101-110, 2000.

Stephenson, D. B.: Use of the "Odds Ratio" for Diagnosing Forecast Skill, Weather Forecast., 15, 221-232, doi:10.1175/15200434(2000)015<0221:UOTORF>2.0.CO;2, 2000.

Tartaglione, N.: Relationship between Precipitation Forecast Errors and Skill Scores of Dichotomous Forecasts, Weather Forecast., 25, 355-365, doi:10.1175/2009WAF2222211.1, 2010.

Wacker, S., Gröbner, J., Zysset, C., Diener, L., Tzoumanikas, P., Kazantzidis, A., Vuilleumier, L., Stöckli, R., Nyeki, S., and Kämpfer, N.: Cloud observations in Switzerland using hemispherical sky cameras, J. Geophys. Res. Atmos., 120, 695-707, doi:10.1002/2014JD022643, 2015.
Winker, D. M., Pelon, J., Coakley, J. A., Ackerman, S. A., Charlson, R. J., Colarco, P. R., Flamant, P., Fu, Q., Hoff, R. M., Kittaka, C., Kubar, T. L., Le Treut, H., McCormick, M. P., Mégie, G., Poole, L., Powell, K., Trepte, C., Vaughan, M. A., and Wielicki, B. A.: The CALIPSO Mission: A Global 3D View of Aerosols and Clouds, B. Am. Meteor. Soc., 91, 1211-1229, doi:10.1175/2010BAMS3009.1, 2010.

Wolters, E., Deneke, H., van den Hurk, B., Meirink, J., and Roebeling, R.: Broken and inhomogeneous cloud impact on satellite cloud particle effective radius and cloud-phase retrievals, J. Geophys. Res., 115, doi:10.1029/2009JD012205, 2010.

Woodcock, F.: The Evaluation of Yes/No Forecasts for Scientific and Administrative Purposes, Mon. Weather Rev., 104, 1209-1214, doi:10.1175/15200493(1976)104<1209:TEOYFF>2.0.CO;2, 1976.

$\mathrm{Wu}, \mathrm{Q} .:$ A correlation-relaxation-labeling framework for computing optical flow: Template matching from a new perspective, IEEE Trans. Pattern Anal. Mach. Intell., 17, 843-853, 1995.

Wu, Q., McNeill, S., and Pairman, D.: Correlation and relaxation labelling: An experimental investigation on fast algorithms, Int. J. Remote Sens., 18, 651-662, 1997.

Zinner, T., Mannstein, H., and Tafferner, A.: Cb-TRAM: Tracking and monitoring severe convection from onset over rapid development to mature phase using multi-channel Meteosat8 SEVIRI data, Meteorol. Atmos. Phys., 101, 191-210, doi:10.1007/s00703-008-0290-y, 2008.

Zinner, T., Forster, C., de Coning, E., and Betz, H.-D.: Validation of the Meteosat storm detection and nowcasting system Cb-TRAM with lightning network data - Europe and South Africa, Atmos. Meas. Tech., 6, 1567-1583, doi:10.5194/amt-6-1567-2013, 2013. 